\title{
Novel Layered Double Hydroxides-Hydroxyapatite/Gelatin Bone Tissue Engineering Scaffolds: Fabrication, Characterization, and In Vivo Study
}

\author{
Fateme Fayyazbakhsh ${ }^{1}$, Mehran Solati-Hashjin ${ }^{1,2^{*}}$, Abbas Keshtkar $^{3}$ \\ Mohammad Ali Shokrgozar ${ }^{4}$, Mohammad Mehdi Dehghan ${ }^{5,6}$, Bagher Larijani ${ }^{7}$, \\ ${ }^{1}$ Biomedical Engineering Faculty, Amirkabir University of Technology, Tehran, Iran \\ ${ }^{2}$ Biomaterials Center of Excellence, Amirkabir University of Technology, Tehran, Iran \\ ${ }^{3}$ Department of Health Sciences Education Development, School of Public Health, Tehran University \\ of Medical Sciences, Tehran, Iran \\ ${ }^{4}$ National Cell Bank of Iran, Pasteur Institute of Iran, Tehran, Iran \\ ${ }^{5}$ Department of Surgery and Radiology, Faculty of Veterinary Medicine, University of Tehran, \\ Tehran, Iran \\ ${ }^{6}$ Institute of Biomedical Research, University of Tehran, Tehran, Iran \\ ${ }^{7}$ Endocrinology and Metabolism Research Center, Endocrinology and Metabolism Clinical Sciences \\ Institute, Tehran University of Medical Sciences, Tehran, Iran
}

\section{Abstract}

Developing porous biodegradable scaffolds through simple methods is one of the main approaches of bone tissue engineering (BTE). In this work, a novel BTE composite containing layered double hydroxides (LDH), hydroxyapatite (HA) and gelatin (GEL) was fabricated using co-precipitation and solvent-casting methods. Physiochemical characterizations showed that the chemical composition and microstructure of the scaffolds were similar to the natural spongy bone. Interconnected macropores ranging over 100 to $600 \mu \mathrm{m}$ were observed for both scaffolds while the porosity of $90 \pm 0.12 \%$ and $92.11 \pm 0.15 \%$, as well as, Young's modulus of $19.8 \pm 0.41$ and $12.5 \pm 0.35 \mathrm{GPa}$ were reported for LDH/GEL and LDH$\mathrm{HA} / \mathrm{GEL}$ scaffolds, respectively. The scaffolds were degraded in deionized water after a month. The SEM images revealed that between two scaffolds, the LDH-HA/GEL with needlelike secondary HA crystals showed better bioactivity. According to the alkaline phosphatase activity and Alizarin red staining results, LDH-HA/GEL scaffolds demonstrated better bonespecific activities comparing to $\mathrm{LDH} / \mathrm{Gel}$ scaffold as well as control sample $(\mathrm{P}<0.05)$. The rabbit adipose stem cells (ASCS) were extracted and cultured, then seeded on the LDHHA/GEL scaffolds after confluence. Three groups of six adult rabbits were prepared: the scaffold+ASCs group, the empty scaffold group and the control group. The critical defects were made on the left radius and the scaffolds with or without ASCs were implanted there while the control group was left without any treatment. All animals were sacrificed after 12 weeks. Histomorphometric results showed that the regeneration of defects was accelerated by scaffold implantation but ASC-seeding significantly improved the quality of new bone formation $(P<0.05)$. The results confirmed the good performance of LDH-HA/GEL scaffold to induce bone regeneration. 


\section{Key words}

Layered double hydroxides, Hydroxyapatite, Gelatin, Biomimetic, In vivo, Adipose stem cell

\section{Introduction}

In recent years, many scholars have paid great attention to the design and preparation of novel materials for bone tissue engineering applications [1]. Bone is a natural nanocomposite containing collagen, non-collagenous proteins and hydroxyapatite (HA), whose complex structure is responsible for both comprehensive and shear strength [2], therefore, mimic this structure to design synthetic scaffolds can be a fair approach for bone regeneration. Generally, the main components of these composite scaffolds are an inorganic bioactive phase and a biocompatible polymeric matrix to prepare an optimized tradeoff between bone healing capacity, remodeling facility, and mechanical strength [3-6]. Obviously, the scaffolds' chemical composition as well as structural characteristics are vital to obtain successful post-implantation outcomes. Additionally, to avoid stress shielding and bone resorption, their mechanical properties should be in the same range of the surrounding tissue. Moreover, since the scaffolds are temporary degradable supports, it is essential to evaluate the biocompatibility of degradation products and monitor new bone formation in the long term $[7,8]$.

Furthermore, a porous scaffold should support cellular activities such as adhesion, growth, and differentiation, and also allow osteocytes to seed into interconnected pores to integrate with the surrounding bone histologically. Microporosity with a pore size less than $10 \mu \mathrm{m}$ is needed to obtain a fine capillary network and cell-scaffold interactions. Macroporosity with a pore size in the range of 150-900 $\mu \mathrm{m}$ allows for osteocytes seeding into scaffolds [9].

According to the biomimetic strategies in many researches, HA and collagen have been utilized to fabricate artificial temporary bone substitutes (i.e. scaffolds) [4]. Many bioactive ceramics such as calcium phosphates, aluminosillicates, layered double hydroxides (LDHs) and bioglasses, have been developed for BTE and/or traditional dental/orthopedic applications [10-12]. On the other hand, similar amino acid sequencing, and the spontaneous capacity of transformation to the necessary type of collagen in the body, make gelatin (GEL) an appropriate choice for the scaffold matrix. GEL as a degradable biopolymer derived from hydrolyzed collagen, cures the immunogenicity and pathogen transmission issues of collagen as well as batch-to-batch differences and limited accessibility. Despite these advantages, GEL has poor mechanical properties and also a high degree of solubility leads to rapid degradation [13]. A promising approach to overcome these limitations, and also meet the biomimetic suggestion is to reinforce it with bioactive bioceramics to achieve an optimal tradeoff between bioactive and chemical behaviors, and physiomechanical properties [1,5]. Furthermore, some researchers reported the effect of GEL in bioactivity through modulating the diffusion of calcium ions that leads to enhance biomineralization specially when impregnated by various inorganic agents [14-16].

$\mathrm{HA}$ is a widely used bioactive phase in BTE scaffolds. It has a great ability to develop the bone in-growth, and to bond strongly with natural healthy tissues [17]. The LDHs, also known as anionic clays, belong to the class of layered ceramics that have recently gained 
much attention. The anionic exchange capacity, and the ability of organic/inorganic anionic intercalation make them a great inorganic carrier [18-20] (Figure 2-c).

In this study, we aimed to fabricate a novel BTE composite scaffold to facilitate the bone healing process. First, the powders of pure LDH and LDH-HA composite were synthetized via co-precipitation method. Then biomimetic porous scaffolds were fabricated through casting method combined with lamination and freeze dry techniques. Afterwards, the resulted scaffolds were studied to evaluate the structural and mechanical similarity with natural bone. Subsequently, in vitro and in vivo studies were run to declare the cellular/tissue responses and biocompatibility of the scaffolds.

\section{Materials and methods}

\subsection{Powder synthesis}

In this study, LDH and a novel LDH-HA nanocomposite were utilized as initial powders. The composite specimen containing $75 \% \mathrm{LDH}$ and $25 \% \mathrm{HA}$ was synthetized through combining the HA synthesis route [21] with the LDH's [20] simultaneously in defined stoichiometric ratios - as described in our earlier work [19]. In details, the precursors Calcium nitrate (Merck, Germany) and Aluminum nitrate (Merck, Germany) were mixed and stirred in a reactor (Ashke Shishe, Iran). Then the diammonium hydrogen phosphate solution (Merck, Germany) was added to the reactor drop-wise. After that, sodium hydroxide $1 \mathrm{M}$ solution (Merck, Germany) was added drop-wise to regulate the $\mathrm{pH}$ at an alkaline range $(\mathrm{pH}=11)$ under a nitrogen atmosphere at room temperature. Then the suspension underwent a $22 \mathrm{~h}$ aging, then was washed and dried via an air oven (PTN International) for $12 \mathrm{~h}$ at $60^{\circ} \mathrm{C}$.

\subsection{Scaffolding}

To fabricate porous scaffolds, a method combining solvent casting, and lamination techniques were used according to Azami et al. [17]. GEL (Merck, Germany) was used as the main matrix of the porous scaffold. After obtaining a homogenous $10 \%(\mathrm{w} / \mathrm{v})$ aqueous $\mathrm{GEL}$ solution at $39 \pm 1{ }^{\circ} \mathrm{C}$, the powder was added and stirred for $1 \mathrm{~h}$ in 450-600 RPM (GEL-powder ratio: 60:40). Afterward, the slurry was homogenized in an ultrasonic bath (Sonoswiss, Switzerland) for $10 \mathrm{~m}$, then, the slurry was spanned in a mold and transferred immediately to a $-22^{\circ} \mathrm{C}$ freezer for $24 \mathrm{~h}$ and then freeze dried for $24 \mathrm{~h}$. The resulted sheets were cut to $3 \mathrm{~mm}$ diameter circular shape, and then attached together with $10 \%$ GEL solution as glue to form a cylindrical structure. Afterward, the porous scaffolds were immersed in a $1 \%$ glutar aldehyde (GA), (Sigma Aldrich, US) solution to enhance the mechanical strength of the polymeric network due to crosslinks ( $24 \mathrm{~h}$ on a roller mixer). After that, the scaffolds were washed with graded ethanols (Merck, Germany) to avoid the presence of any residue of GA. After drying at room temperature, the scaffolds were carefully filed and trimmed to obtain an exact circular cross section with the height of $15 \mathrm{~mm}$ and $10 \mathrm{~mm}$, for biological evaluations and mechanical testing, respectively. 


\subsection{Characterization}

\subsubsection{Structural analysis}

The synthesized powders were examined by a Siemens-Bruker D5000 diffractometer (AX $\mathrm{GmbH}$ Karlsruhe, Germany) with $40 \mathrm{kV}$ voltage and $40 \mathrm{~mA}$ current setting, using Cu-K $\alpha$ radiation $\left(1.54051^{\circ} \mathrm{A}\right)$. For qualitative analysis, XRD patterns were recorded in the interval $4 \leq 2 \theta \leq 70$ at a scan speed of $28 / \mathrm{min}$ (step size of 0.028 and step time $1 \mathrm{~s}$ ).

The chemical structures of scaffolds were studied by Fourier transform infrared (FTIR) of Perkin Elmer Corp. spectrometer (Norwalk, US). The samples were scanned at the range of $400-4000 \mathrm{~cm}^{-1}$ wavenumbers with a $4 \mathrm{~cm}^{-1}$ resolution at the scan speed of $23 \mathrm{scan} / \mathrm{min}$.

The samples were first coated with a thin layer of gold using a sputter coater (EMITECH, England) and then were investigated semi-quantitatively by scanning electron microscopy (SEM, Philips, Netherlands) equipped with an Energy Dispersive Spectrometer (EDS), operating at the acceleration voltage of $15 \mathrm{kV}$. The elemental ratios were obtained through the distribution maps of the elements $\mathrm{Ca}, \mathrm{Al}$, and $\mathrm{P}$ from three random points using ImageJ software. In vitro cellular activity (cell morphology, cell viability, and cell behavior analysis), in vivo new bone formation, and the primary observation of scaffolds were captured by light microscopy (KS400, Zeiss, Switzerland).

\subsubsection{Porosimetry}

The porosity of scaffolds was measured according to Archimedes' principle using ethanol as solvent based on Kim et al. [22]. Dry weights of all the scaffolds were measured using a 4digit digital scale (Sartorius, Germany). The pores' size and shape of the scaffolds were observed by SEM and quantitatively measured via Image J software.

\subsubsection{Degradation}

Three pieces of each of the scaffolds were initially weighted accurately and then immersed in deionized water. To avoid unnecessary complexity, the degradation behavior, mass loss and water uptake were studied in deionized water at this stage. The $\mathrm{pH}$ and weight of each sample were measured in determined time points. The curves of mean weight and $\mathrm{pH}$ concentration were plotted versus time.

\subsubsection{Mechanical properties}

The ultimate compressive strength, Young's modulus and stiffness of scaffolds were measured at room temperature. The mechanical testing setup was adjusted on $10^{-2} \mathrm{~N}$ and $10^{-6} \mathrm{~m}$ axial force resolution via a dynamic mechanical testing machine (Zwick/Roell, Germany) using the Toolkit1998 software to get results with a default constant displacement with the rate of $1 \mathrm{~mm} / \mathrm{min}$. As mentioned before, the scaffolds were shaped to cylinders and filed to $3 \mathrm{~mm}$ in diameter and $6 \mathrm{~mm}$ in height based on the ASTM F 2150-07 standards. Additionally, in order to meet statistical issues, all of the tests have been repeated three times and the average and standard deviations (SD) were measured and plotted. 


\subsection{In vitro evaluations}

\subsubsection{Bioactivity evaluation}

Simulated Body Fluid (SBF) was prepared based on the Kokubo et al. recipe, and all materials were prepared from Merck Inc. All samples were soaked in SBF for 21d to study the secondary HA formation as an indicator of bioactivity [23].

\subsubsection{Indirect extraction}

In this study, we used the G-292 cell line (NCBI, Iran) to investigate the biological properties of the samples because it line provides similar conditions to normal osteoblast cells due to less differentiation [24]. G-292s were cultured in RPMI (Gibco, US) fortified by $10 \%$ fetal bovine serum (FBS - Seromed, Germany), $100 \mathrm{U} / \mathrm{ml}$ penicillin (Sigma Aldrich, US) and 100 $\mu \mathrm{g} / \mathrm{ml}$ streptomycin (Sigma Aldrich, US) and were incubated at $37^{\circ} \mathrm{C}$ in a humidified atmosphere with $5 \% \quad \mathrm{CO}_{2}$. Cellular activities were appraised using the extracts of powder/scaffold that were prepared according to the ISO-10993 protocols. $0.1 \mathrm{~g}$ of each sample per $1 \mathrm{ml}$ of culture media was incubated. At the end of 3,7 and 14 days, the medias were collected and filtered for use in different in vitro tests. Pure culture media under similar conditions was considered as the control sample.

\subsubsection{MTT assay}

The proliferation rate of cells in the extracts of powders and scaffolds was estimated by conducting the MTT (3-(4,5-dimethylthiazol-2-yl)-2,5-diphenyltetrazolium bromide- Sigma, US) assay. In summary, first, $10^{4} \mathrm{G}-292$ cells/well were cultured into a 96 -well plate (Nunc, Denmark) with $100 \mu$ media. After $24 \mathrm{~h}$, the old media was replaced by $90 \mu \mathrm{l}$ sample extracts plus $10 \mu \mathrm{l} \mathrm{FBS}$ and removed after $24 \mathrm{~h}$ and then $100 \mu \mathrm{l}$ of $0.5 \mathrm{M}$ MTT solution was added to each well. After $4 \mathrm{~h}, 100 \mu \mathrm{l}$ of isopropanol (Sigma, US) was added to each well for $30 \mathrm{~min}$ to form the dark blue formazan crystals. Finally, optical density (OD) of formazan was measured at 545nm using a scanning multiwall ELISA reader (STAT FAX 2100, US) as in indicator of cell viability. The obtained data were normalized considering the control sample.

\subsubsection{Alkaline phosphatase activity}

The functional activity of G-292s was investigated by detecting the activity of the ALP enzyme. First, $10^{5}$ cells/well were cultured in a 24 -well plate and after $3 \mathrm{~h}$, the old media was replaced by new media containing $80 \%$ extracts, $10 \%$ RPMI and $10 \%$ FBS. Then, the medias were collected after 7 and 14 days. Total ALP amounts were measured calorimetrically using the Autoanalyzer (Hitachi 902, Japan) and ALP assay kit (ParsAzmoun, Iran) based on the hydrolysis of $p$-nitrophenyl phosphate to form the colored $p$-nitrophenol in the presence of ALP.

\subsubsection{Alizarin red staining}

The presence of deposited calcium extracted by G-292 cells was determined by the Alizarin red staining (ARS) method. First, $10^{6} \mathrm{G}-292$ cells were cultured in a 6-well plate and after $3 \mathrm{~h}$, ordinary culture media was removed and replaced by new media containing $80 \%$ extracts, $10 \% \mathrm{RPMI}$ and $10 \% \mathrm{FBS}$. After $3 \mathrm{~d}$, the old media was removed and rinsed three times with 
saline serum (0.9\%). Then, the cells were fixed by GA (1\%) for $10 \mathrm{~m}$ and afterward stained with $A R(2 \%, \mathrm{pH} 4.2)$ for $45 \mathrm{~m}$ at room temperature. The cells were washed with saline serum before OM observation. Also, this test was conducted on ASCs under similar conditions to study the biomineralization and differentiation processes.

\subsection{In vivo study}

\subsubsection{Animal groups}

The animal study was carried out in compliance with the procedures and approval of the Institutional Animal Care and Use Committee of Tehran University of Medical Sciences and conformed to ISO 10993 codes of ethics for animal testing.

18 mature New Zealand white male rabbits $(1.5-2.5 \mathrm{~kg}$ ) were housed with free access to food and water. They were divided into 3 groups of A-Cell-scaffold group: critical bone defect with adipose ASC loaded on LDH-HA scaffold, B- Scaffold group: critical bone defect with LDH-HA scaffold and C- Control group: critical bone defect left without any treatment. To meet the ethical issues, the in vivo tests were run only for the optimized scaffold (i.e. LDH-HA-GEL scaffold) to use the least possible number of animals.

\subsubsection{ASC extraction}

Fat tissue was sampled from the intrascapular area under general anesthesia. Then, fat tissues were washed and cut to remove the blood cells, vessels and fibrosis tissues. Afterward, collagenase type I solution $(0.25 \mathrm{M})$ was added to the samples in a shaker incubator at $37^{\circ} \mathrm{C}$ and $250 \mathrm{rpm}$ for $1 \mathrm{~h}$. Then $20 \%$ FBS was added to the samples to stop collagenase activity. After washing the samples, they were cultured in adipogenic culture media for 14 days to identify the type of extracted stem cells. The oil-red-O staining technique was used to evaluate the specific function of differentiated adipose stem cells [25]. Afterward, the obtained ASCs were cultured in osteogenic media -containing ascorbic acid, $\beta$-glicerophosphate and dexamethasone- for $21 \mathrm{~d}$.

\subsubsection{Implantation}

All the scaffolds with the height of $15 \mathrm{~mm}$ and diameter of $5 \mathrm{~mm}$ and then sterilized using ethylene oxide for $10 \mathrm{~h}$ prior to the implantation. Afterward, ASCs were seeded onto the scaffolds. The animals were anesthetized by intramuscular injection of $75 \mathrm{mg} / \mathrm{kg}$ ketamine hydrochloride (Alfasan, Holland) and $10 \mathrm{mg} / \mathrm{kg}$ xylazine (Bayer, Canada) and the anesthesia was continued using isoflurane $1.5 \%$ after endotracheal intubation. The two groups of scaffolds (with/without ASCs) were implanted into a $15 \mathrm{~mm}$ critical defect on the left radius bone (in the control samples the defects were left without any treatment). The new bone formation was observed via radiography at week 0 (after implantation), 4, 8 and 12. In order to measure the density of newly formed bone, an aluminum graded phantom ranging from 0.5 to $4 \mathrm{~mm}$ was located close to the defect area (The implantation process was shown in figure 1). Then the quantitative amount of new formed bone was calculated using ImageJ software. At the end of week 12, the animals were sacrificed via overdosed intravenous injection of sodium pentobarbital (Alfasan, Holland). Afterward, the radius bone and its 
surrounding tissues were dissected from the soft tissues and the tissue radiographs were captured.

For histological observation, the tissues were immediately fixed with $10 \%$ formalin solution for $24 \mathrm{~h}$ and decalcified with 14\% EDTA for 2 weeks. After paraffin embedding, cutting and mounting on the slides, the sections were stained with hematoxylin and eosin (H\&E) prior to OM observation. In order to determine the areas that were occupied by newly formed bone (cortical/spongy), connective tissue, osteoid and scaffold residues, the computer software Sigma Scan Pro was employed.

\subsection{Statistical Analysis}

According to ISO 10993, all experiments including mechanical tests, degradation, MTT, ALP, and histomorphometry were performed in 3-10 replications. Quantitative data were reported as means standard deviations. Microsoft Excel 2016 software was utilized for the variance analysis of repeated measurements (i.e. ttest) with the consideration of statistically significant for $\mathrm{P}<0.05$.

\section{Results and Discussion}

\subsection{Structure}

Figure 2-a represents the micrograph of synthesized LDH powder. The spherical morphology and nearly homogeneous particle size can be observed in SEM images. The morphology, the high degree of agglomeration, and the average particle size of $50 \mathrm{~nm}$ can be a result of $22 \mathrm{~h}$ aging, that leads to particle agglomeration and high surface energy [18]. This is the key to justify the significant surface reactions and bioactivity. In figure 2-d the microstructure of the LDH-scaffold was shown. Non-uniform interconnected pores can be seen in two main classes: the smaller pores with the average size of 20 to $50 \mathrm{~nm}$ with spherical shape, thicker walls and more regular shape. The other one showed the bigger pores in a range of 200$600 \mathrm{~nm}$-suitable for osteocyte seeding [9]- with less regularity in shape and inhomogeneous thinner walls. The seeded osteoblast on the scaffold can be seen in figure 2-g. In addition, specific cellular behaviors can be observed: well spread seems to migrate to the left side, ECM, philopedias formation and also the cell anchorage on the surface. Qualitatively, all of these evidences show that the cells feel comfortable on the surface of the LDH scaffold.

Figure 2-b represents the microstructure of LDH-HA nanocomposite powder. With due attention to the same condition of synthesis in both LDH and LDH-HA powders, less particle size regularity and the higher degree of agglomeration of this specimen can be the result of higher surface energy that can lead to more surface interactions and bioactivity. Due to the high degree of agglomeration, the morphology of particles cannot be exactly recognized, but it can be approximately stated as the spherical shape. The microstructure of the LDH-HA scaffold was shown in figure 2-e. The more homogeneous interconnected pores with more regularity in wall thickness and pore sizes of $20-40 \mathrm{~nm}$ and $100-300 \mathrm{~nm}$ can be observed, respectively. The satisfying shape similarity to the natural spongy bone - Figure 2-f - can be 
a strong cause for selecting this nanocomposite as the BTE scaffold. Also, more cell expansion can be a result of the presence of HA and more similarity to bone composition. This similarity and also appropriate electrostatic interaction, can be a strong clue for the physical signaling factor to motivate cells to migrate on the scaffold surface or to seed into the pores.

The cell-surface interaction of this specimen was shown in figure 2-h. A finer cell spreading and higher degree of ECM polymerization in comparison to the LDH scaffold can be observed. Also, it was demonstrated that this specimen provides a better culture bed for cell communication and routine cellular activities like adhesion, dividing, replication and growth as it can be easily found from the comparison between the two 5 um cells earlydivided cells at the right side and $104 \mathrm{~m}$ mature cell at the left side. Moreover, it was obvious that the cells were growing due to the rising size of these cells from 5ym to $104 \mathrm{~m}$. According to the orientation of the philopedias, the better synthesis of ECM on the scaffold surface and cell migration towards the pores can be the result of appropriate surface physiochemical properties. Figure 2-i represents the surface topography of the LDH-HA scaffold that provides a fine bed for the production of cellular colonies on the surface.

$\mathrm{XRD}$ is a powerful technique to study the structure of crystalline materials such as HA and $\mathrm{LDH}$. The lattice parameter $d$ of $\mathrm{LDH}$, which reflects the interlayer spacing as a great capacity of drug delivery applications and also solubility, diffusion and degradation rate, can be calculated directly through Bragg's Law. The X-ray diffraction patterns of the synthesized LDH and LDH-HA powder specimens were plotted in figure 3-a. According to these patterns, new phases were formed in the LDH-HA pattern and also, a decrease in LDH peaks coinciding with the presence of HA peaks in this specimen was observed. According to the JCPDS reference carts for HA [26], and also LDH characterization articles $[18,20]$, the presence of $L D H$ and $H A$ is obvious. Based on the literature, the XRD pattern of LDH represents a typical well-ordered structure with a basal spacing (plain do03) of $7.77 \AA$ [27]. This value is marginally larger than the calculated value of the interlayer space in the LDHHA specimen. The LDH-HA also gives two small sharp peeks at about $25^{\circ}$ caused by the intermediate phases. The presence of HA in this specimen leads to a rise in ordered diffraction peaks at lower angles (i.e. a little shift to left-side), indicating that some anions have entered the interlayer space of LDH. The pure LDH specimen shows a higher degree of crystallinity than the LDH-HA specimen, because it gives sharper peaks with higher intensity and lower width, while LDH-HA simply appears at broad peaks. This confirms the finer particles as represented in Figure 2-a and 2-b. The $d_{003}$ value of LDH-HA is $8.265418 \AA$, that is $0.4897 \AA$ lower than the LDH specimen. Considering the lower radius of carbonate compared to nitrate $(0.189 \AA$ and $0.200 \AA$ respectively), it can be easily found that carbonate groups have a greater chance to intercalate in the LDH interlayer space in the LDH-HA specimen. Also, in this specimen the indication peek of HA is wider than a usual HA XRD pattern, which can be the result of carbonate apatite formation [28].

The ratio of LDH to HA was derived by using Eq. 5 and also the lattice parameters for the HA phase and LDH phase were calculated by using Eq. 6 and 7, respectively [18, 29]. 
As it was shown in table 1 , the lattice parameter of $\mathrm{HA}$ remained constant because the HA lattice did not change, but the lattice parameters of LDH were changed. Hence, these parameters of LDH were sensitive to the other ions in the LDH-HA specimen, and the $\mathrm{C}$ parameter decreased which may be a result of more electrostatic attraction between hydroxyl groups and phosphate bonding than nitrate.

$$
\begin{gathered}
R I R=\frac{I_{L D H}}{I_{L D H}+I_{H A}} \text { (Equation 1) } \\
c_{H A}=l * d \quad a=d * \sqrt{\frac{4}{3} *\left(h^{2}+h k+k^{2}\right)} \quad \text { (Equation 2) } \\
c_{L D H}=d_{003}+2 d_{006}+3 d_{009} \quad d_{\text {average }}=\frac{c}{3} \quad a=2 d_{110} \quad \text { (Equation 3) }
\end{gathered}
$$

The change in $d$-spacing can be a result of phosphate ion separation from HA and intercalation between LDH interlayers. Maybe some of the initial crystals were dissolved and rearranged and plain coherency occurred between shared $\mathrm{Ca}$ ions of both crystals.

The elemental analysis using EDS was performed to confirm the structure of synthetized LDH and LDH-HA (table 1). According to previous researches, the stoichiometric ratio of $\mathrm{Ca} / \mathrm{P}$ for $\mathrm{HA}$ and $\mathrm{Ca} / \mathrm{Al}$ for $\mathrm{LDH}$ (i.e. divalent metal to trivalent metal) have been reported 1.67 and 2, respectively [30, 31]. The $\mathrm{Ca} / \mathrm{Al}$ ratio was reported 2.02 in $\mathrm{LDH}$ sample which is close to the stoichiometric ratio. The LDH-HA specimen as a biphasic ceramic which is supposed to contain $75 \% \mathrm{LDH}$ and $25 \% \mathrm{HA}$, presented the $\mathrm{Ca} / \mathrm{AL}$ value of 2.54 that contains the amount of $\mathrm{Ca}$ ions in both LDH and HA networks (i.e $\frac{C a_{L D H}+C a_{H A}}{A l_{L D H}}$ ). Likewise, the $\mathrm{Ca} / \mathrm{P}$ value was reported 7.65 (i.e $\frac{C a_{L D H}+C a_{H A}}{P_{H A}}$ ), that can be obtained if the $C a_{H A} / P_{H A}$ value was considered close to 1.67 with $25 \%$ weigh of the composite. That confirms the successful synthesis in accordance with XRD patterns.

The FTIR spectra of specimens are plotted in figure 3b. The main bonds of HA [32] and LDH [33] and also, variant amide groups of GEL [34,35] were marked in this plot, despite the overlaps. The phosphate peeks as an indicator of the HA phase were increased in the LDHHA specimen and the nitrate peaks as an indicator of LDH showed a decrease in this specimen compared to the LDH scaffold. Another result of ion exchange of phosphate groups, is to have more affinity to the bond with brocit layers than nitrate groups [18], thus the phosphate groups of HA are rearranged in the interlayer space of LDH and took part in both phases. The peaks of amide groups are sharper and show more depth in the LDH-HA group, which can be the result of lower crystallinity and more space to accommodate the network of GEL. On the other hand, sharper peaks of the water-related bonds show the higher capacity of swelling in this specimen. This can lead to the more homogenous microstructure of scaffold, as it can be observed in Figure 2-e versus 2-d.

Considering the hydrolysis-sensitive bands in the FTIR plot such as amide groups, carbonate/nitrate-OH or $\mathrm{Ca}-\mathrm{OH}$, it can be predicted that the LDH-HA scaffold degrades faster. 
The decrease of $\mathrm{H}_{2} \mathrm{O}$ peaks in the pure LDH scaffold indicates the reduction of interlayer distance (parameter c) and also more crystallinity in accordance with XRD patterns. Likewise, the accommodation of the interlayer space with phosphate groups -with a larger anionic radius- can be another reason for more voids to appear and situate more waters in the LDH-HA scaffold. Also, the decrease of H-bonds in the pure LDH scaffold demonstrates that the larger anions were intercalated in the interlayer space of the LDH-HA specimen similar to phosphate with more charge and larger anionic radius.

For both specimens, there is an intense and broad bond at $\sim 3410 \mathrm{~cm}^{-1}$ related to the Al-OH stretching vibrations. The width of this bond is due to $\mathrm{H}$-bonds that has a higher intensity in the LDH-HA specimen. Therefore, it was illustrated that more hydrogen bonds were formed in this specimen due to phosphate groups and also more porosity which leads to more swelling capacity. According to the layered structure of LDHs -positively charged sheets with absorbed anions in the interlayer space- the strong band at $3410 \mathrm{~cm}^{-1}$ is responsible for Ca$\mathrm{OH}$ stretching mode whereas the $3575 \mathrm{~cm}^{-1}$ shoulder on the higher wavenumbers may be attributed to $\mathrm{Al}-\mathrm{OH}$ vibrations [33, 36].

An adsorption bond at $1350 \mathrm{~cm}^{-1}$ is responsible for the $\mathrm{CO}_{3}$ stretching mode placed in the LDH interlayer. The large increase of the peak intensity at $1350 \mathrm{~cm}^{-1}$ in the spectra of the LDH-HA scaffold reflects the replacement of interlayer nitrate by carbonate which occurred more in this specimen compared to that of pure LDH. This is in accordance with the establishment of more water molecules due to the greater affinity of water to carbonate more than nitrate. The complex region in the low wave numbers is assigned to a complex profile showing multiple bands of amide groups of gelatin.

The sharp bond at $3400 \mathrm{~cm}^{-1}$ is attributed to the water stretching vibration. The complex bonds between 2500 and $3200 \mathrm{~cm}^{-1}$ correspond to the amide groups of gelatin. The significant increase of these peaks in the LDH-HA scaffold indicates the greater water absorption that leads to more swelling of gelatin because of the more void presence due to less crystallinity. Therefore, it is expected that this scaffold has a faster degradation rate. On the other hand, the stronger peaks of amide groups in this scaffold can be a result of more electrostatic interaction between mineral/organic phases as a consequence of less crystallinity according to the XRD patterns.

The bond at $1337 \mathrm{~cm}^{-1}$ is attributed to the vibration mode of proline side chains in GEL. That is one of the bonds of the Amide A-B complex. The amide A bond rising from 3270 to 3370 $\mathrm{cm}^{-1}$ related to the $\mathrm{N}-\mathrm{H}$ stretching mode is responsible for cross linking. Also, the bond at $\sim 2920 \mathrm{~cm}^{-1} 1$ is attributed to $\mathrm{C}-\mathrm{H}$ stretching in amide $\mathrm{B}$. The bond at $1670-1650 \mathrm{~cm}^{-1}$ attributed to $\mathrm{C}=\mathrm{O}$ stretching confirms the presence of the amide $\mathrm{I}$ group and the $\mathrm{N}-\mathrm{H}$ deformation at $1500-1550 \mathrm{~cm}^{-1}$ attributed to the amide II, verifies the formation of the amide II group [37]. According to the studies, it can be stated that both scaffolds adopt a $\alpha$ helical configuration between mineral/GEL phases and this is in accordance with the appearance of amide II at $\sim 1540 \mathrm{~cm}^{-1}$ approximately [35].

The shift of the Amide groups of LDH-HA/GEL scaffold can be an evidence of the formation of a chemical bond between carboxyl ions in GEL and HA phases -due to the presence of 
phosphate groups of the HA part. The stronger peaks in this region for the LDH-HA/GEL scaffold demonstrate the more electrostatic attraction between mineral/organic phases. On the other hand, the covalent bonds are formed between $\mathrm{Ca}^{2+}$ ions and $-\mathrm{COO}^{-}$terminals of amide groups. In addition, the cross-linking process reduces the distance between the ceramic-GEL complex less than the critical length and therefore, the possibility of formation of bonds between $\mathrm{Ca}^{2+}$ cations and R-COO- anions will be increased [37, 38].

As was mentioned, GA was used as a cross linking agent to optimize the mechanical properties, but many researchers reported the high toxic effect of this material [39]. The main peak of this hazardous material can be observed at 1710 to $1730 \mathrm{~cm}^{-1}$ attributed to free-aldehydic bonds [40]. A weak peak at this range can be seen in both scaffolds but it overlaps with the $\mathrm{H}_{2} \mathrm{O}$ bending peak, thus we cannot strictly comment on the traces of GA.

\subsection{Physiochemical properties}

The physiomechanical properties of the scaffold versus natural spongy and cortical bone were reported in Table 2 . The stress-strain curves of both scaffolds and cortical and also, spongy bone were plotted in figure 3-c. According to these, the density and mechanical properties of both scaffolds are reported close to the natural spongy bone, therefore, it is expected that these scaffolds will prevent the stress shielding in vivo. Moreover, the density of the LDH/GEL scaffold was $2 \%$ higher than the LDH-HA/GEL scaffold but compared to natural spongy bone, this difference can be considered insignificant. Additionally, the porosity of this specimen is about $2 \%$ more than the pure LDH /GEL scaffold. The Young's modulus was reported as $19.8 \pm 0.41$ and $12.5 \pm 0.35 \mathrm{GPa}$ for the pure LDH/GEL and LDH$\mathrm{HA} / \mathrm{GEL}$ scaffold, respectively. This significant difference $(\mathrm{P}<0.05)$ can be a result of lower crystallinity, lower density, more porosity and also, starting material. According to the inverse relation between $E$ and porosity [41], the higher porosity of the LDH-HA/GEL scaffold can be a strong reason for the lower young modulus of this specimen. The ultimate compressive strength (Fucs) was measured by applying the load until the fracture of scaffolds. The Fucs pure LDH/GEL scaffold was reported more than the other sample. Based on the rule of mixtures [42] the amount of HA in that scaffold can lead to a brittle behavior and lower E, Fucs and yield strength.

In the pure LDH scaffold, the layered structure of LDH can be a cause for a higher young's modulus. The layers are slipping over each other and this leads to more elasticity. On the other hand, the formation of more water molecules between amide groups and also more voids in the structure of the LDH-HA/GEL scaffold, lower the mechanical properties.

Generally, the apparent density of porous materials such as scaffold is defined by the measurement of the volume of open pores that have crucial roles in mechanical strength, permeability, water absorption, surface area and degradation rate. These scaffolds also showed a highly interconnected $3 \mathrm{~d}$ porous network enhancing the nutrients and metabolite transportation through the scaffolds. The homogenous swelling in all of the repetitions of both samples confirms the formation of interconnected pores. On the other hand, the degradation processes of both samples did not include any phase detachment or 
macroscale cleavage which is the reason for the strong adhesion of initial powders to GEL particle detachment can cause future clinical problems such as inflammatory response [43]. The plots of water absorption of scaffolds were shown in figure 4-a. The water absorbance of scaffolds reached the maximum of about $295 \%$ and $415 \%$ of the initial weight after $2 \mathrm{~h}$ for pure LDH/GEL and LDH-HA/GEL scaffolds, respectively. The studies regarding the swelling of GEL and HA/GEL scaffolds reported the ultra-high swelling capacity and the ability of more water entrapment than their initial weight. Also, they reported a quick equilibrium time of swelling that corresponds to the degree of porosity and hydrophilicity [44]. Moreover, the more the interactions between LDH-HA and GEL than pure LDH, the faster the relaxation of GEL chains that leads to an increase in the swelling ratio. As was reported in table 1 , the lattice parameters of both phases of LDH and HA had a significant difference. Obviously, larger lattice parameters can lead to an increase in diffusion rate, solubility and degradation rate. The cumulative lattice parameters in LDH-HA powder is about $23 \%$ more than the pure LDH powder. It is expected to absorb more water in the LDH-HA/GEL scaffold -in accordance with the FTIR spectra. Therefore, this specimen absorbs more water due to more voids of initial powder, more pores of scaffold and also, more amide groups after scaffolding and larger capacity of swelling. After $2 \mathrm{~h}$, descending mild slopes were recorded for both scaffolds. After day 4, a major weight loss has occurred in both scaffolds that indicated the beginning of the degradation process. Afterward, the degradation curves fall with gentle slopes and eventually end at day 32. These curves include two main parts: swelling till day 4 and degradation till day 32 . On the other hand, it should be mentioned that the main degradation mechanism is the hydrolytic mechanism because there is no microorganism or enzyme in the degradation environment.

The degradation process can be a result of GEL degradation in an aqueous environment and release of $\mathrm{OH}^{-}$groups from the ceramic part (both $\mathrm{LDH}$ and $\mathrm{HA}$ ). The greater alkaline condition leads to faster hydrolytic degradation in the LDH-HA/GEL scaffold and the GEL molecular weight decreases faster in this specimen. The graph of changes in $\mathrm{pH}$ were plotted in figure 4-b. At first, GEL provides an acidic environment, then LDH and HA were dissolved and increase $\mathrm{pH}$. Afterward, the GEL degradation speeds up. Since the alkaline power of LDH-HA is more than HA, the degradation of the LDH-HA/GEL scaffold is completed faster.

Obviously, there is a direct relation between molecular weight loss and mass loss of degradable polymers over time, which correspond with tissue regeneration in polymeric scaffolds. The downtrend of $\mathrm{pH}$ is responsible for swelling and water absorbance during ionic exchanges between the GEL and ceramic phase. It is observed that the scopes of both scaffolds are similar up to the first two hours but after that, the $\mathrm{pH}$ of the pure LDH/GEL scaffold dropped to 5.5. This can be a reason for less alkaline power of LDH against LDH-HA and also less concentration of $\mathrm{Ca}^{2+}$ which leads to more concentration of $\mathrm{H}^{+}$and a lower $\mathrm{pH}$. In LDH-HA, HA acts as a wall and resists ion exchange because of the greater alkaline power. On the other hand, according to figure 4-a, the amount of absorbed water in this scaffold is more than LDH/GEL, which is due to the physical attraction between water molecules of the 
GEL network and also water replacement in ceramic lattice voids (i.e. non-chemical reactions) that is more swelling than degradation. In contrast, in the LDH/GEL scaffold, the sharper drop in $\mathrm{pH}$ indicates that the total process is a chemical reaction and degradation occurs more than swelling. It appears that after $16 \mathrm{~h}$, the swelling capacity is completely used and reactions are directed toward shortening the backbone chain of GEL and LDH dissolution (i.e. degradation) due to an increase in $\mathrm{pH}$. After $4 \mathrm{~d}$, the $\mathrm{pH}$ of both scaffolds reach a constant level that can be the result of equilibrium state and eventually the degradation of all masses.

\subsection{Biological evaluations}

In vitro bioactivity tests evaluate the ability of the formation of HA or carbonate HA on the surface of biomaterials, after soaking in SBF which is known to be a supersaturated buffer solution of HA [23]. In figure 5, the SEM images of scaffolds are shown after immersion in $\mathrm{SBF}$, as well as, the interconnected homogeneous pores can be seen, although, the pores' walls gradually started to deform and degrade after soaking in SBF. In these after-SBF scaffolds, needle-like secondary HA crystals can be observed in coincidence with degradation. The diameters and length of HA secondary crystals in the LDH-HA/GEL scaffold are more than the LDH/GEL scaffold. This can be a result of HA presence in this composition and higher $\mathrm{pH}$ that leads to a lower number of crystals on the surface, while yielding larger crystals (i.e. less nucleation and more growth).

It seems that after implantation, a series of more complex reactions occur in comparison with our simple static bioactivity setup. However, the result of this test can predict the in vivo behavior of the scaffolds and also this is a stimulation of the biologic environment [23]. It can be stated that LDH, LDH-HA and GEL could participate in specific reactions, and all of them have their own role in the deposition of the amorphous calcium phosphate and also in the rearrangement stage to form a crystalline HA layer on the scaffold surface, as it can be observed in vitro. Researchers studied the effect of GEL on the formation of secondary HA through modulating the diffusion of calcium or other ions that improves the biomineralization processes [14-16]. This secondary HA will correspond to the strong bonding between the scaffold and surrounding tissue.

According to figures 5-a and 5-d, the mineral deposition on the surfaces of inner pores and the scaffold can be observed. This can transform the scaffold's surface to an optimized bed for cell/tissue interactions.

On the other hand, in the LDH/GEL scaffold, the degradation and wall deformation processes lead to weakening of the mechanical strength. Pores lost their shapes and the crystals of secondary HA grow irregularly and in a preferred direction from the inside to outside of the pores. Another observable event of these images is the formation of small pores that did not exist before SBF. This is a key element of the start of the degradation process. In the LDH-HA scaffold, the greater Ca source and more surface energy lead to secondary HA growth on phosphate groups as templates for preferred growth. Due to the lower concentration of $\mathrm{HA}$, needle like secondary HA crystals from HA zones can be seen. Moreover, these phosphate groups may be shared between the interlayer space of LDH and 
primary HA phase that strengthen the template role of the phosphate hypothesis. Additionally, more arranged secondary HA crystals are greater in diameter and the amount can be seen in this specimen.

The results of G-292 cell viability for the extracts of powders and scaffolds were represented in figure 6-a. As it is plotted in this diagram for 7 day extracts, the scaffolds showed a higher degree of cell viability than the powder samples and also the control sample. This may be a result of the physical properties of powders including particle size and surface energy, leading to reduction in cell viability compared to the control sample. Many researchers have been discussed the correlation between particle size and distribution with in vitro/vivo responses to investigate the possible toxic effect of nanoparticles on various cell sources [45-47]. Therefore, it can be said that the scaffolding process and the presence of GEL enhanced the biocompatibility of initial powders and increase the cell viability because of physical consistency and smoother surface comparing to the powders with more surface charge and less homogeneity in surface topography $[22,48,49]$.

There is no meaningful difference between the LDH-HA scaffold and control sample at the 14-day extract $(P>0.05)$. It can be said that by continuing to raise the extraction time, the cell viability will be improved for powder specimens but the scaffolds do not show a remarkable change. This can be a result of reaching the maximum degree of swelling and the ion exchange that leads to changes in the extraction which reaches equilibrium -in accordance with degradation behavior.

ALP activity as an indicator of osteoconductivity, is one of the main conventional markers of the osteoblastic phenotype expression. Figure 6-b shows the amount of ALP from the gathered culture media after 14 days around the pressed powders and scaffold (direct contact with G-292 cells). Both powders and scaffolds show better ALP activity comparing to the control sample $(P<0.05)$. This can be the result of the diffusion of $\mathrm{Ca}$ ions from the specimens to the culture medias, and higher amount of $\mathrm{Ca}$ around the cells that leads to increase the osteoblastic functionality of G-292 cells and ALP activity. In addition, the scaffolds show a better response because of swelling and hydrogel forming. As mentioned before, the degradation and bioactivity behaviors of these scaffolds promised a better response of scaffold samples. There is no significant difference between the response of LDH-HA/GEL scaffolds after $7 \mathrm{~d}$ and $14 \mathrm{~d}$ and also a slight difference between ALP activity can be seen between $7 d$ and $14 d$ of $L D H / G E L$ scaffolds $(p<0.05)$. This is justified with previous results of swelling and degradation. As it was observed, after day 4, the swelling reaches its threshold, and therefore the ion exchange process reaches equilibrium and afterward the cellular activity slowly rises. Additionally, as reported in the literature, the amount of ALP initially rises and then reaches its maximum level and afterward remains constant because the cells reach confluence, and after a while, decrease during the cell culture process [50]. Thus, it can be said that the cells reached confluence after 14-day culturing.

Additionally, previous studies reported that HA leads to an increase in the ALP activity of osteoblasts as well as the osteoblast response to GEL-HA scaffolds that was reported significantly superior to the GEL in terms of ALP activity levels after 14 days [51]. On the 
other hand, some studies stated that $\mathrm{Ca}$ ions can be directly involved in enhancing the osteoblasts' proliferation and phenotype expression through cell membrane ion transfer, that largely confirms our results [52].

Therefore, the LDH powder and LDH/GEL scaffold show a lower ALP activity in the lack of HA. These results indicate the efficiency of the adding HA (either powder or scaffold) in osteoblastic phenotype expression enhancement. Subsequently, the LDH-HA/GEL scaffold enhances the specific activity of the bone-derived cells.

The quality of Ca mineralization of G-292s and ASCs (only for LDH-HA/GEL scaffold after incubation in osteogenic media) seeded in the scaffolds were shown in figure 7. As shown in this figure, a more obvious positive staining of ARS on LDH-HA/GEL scaffolds was observed than that of HA/GEL scaffolds. The result indicates that the LDH-HA/GEL scaffold facilitates much more Ca mineralization than the LDH/GEL scaffold that is a result of the presence of $\mathrm{HA}$. According to some researches, HA has a key role in the bone mineralization process [53] thus, it can easily be found that the better mineralization in LDH-HA/GEL scaffold is due to the effect of HA composition. It can be stated that as HA acts as a template for secondary HA formation in bioactivity evaluation, it can be a template of in vivo biomineralization. On the other hand, the similarity of GEL and collagen in chemical composition can conduct the biomineralization process so we can obtain better results in scaffolds than the control sample. Additionally, according to the previous results about the LDH composition and structure, it can be an appropriate candidate to influence the biomineralization positively due to the high capacity of ion exchange. In addition, a significant difference can be observed for both scaffolds compared with the control sample. On the other hand, these qualitative comparisons illustrate the greater $\mathrm{Ca}$ deposition in the vicinity of ASCs. Moreover, it proves the differentiation of ASCs to osteocytes. After 14 days of treatment with adipogenic culture media, the cell differentiation into adipocytes was confirmed by OilRed-O staining (figure 7-i). The identification of the resulted stem cell was supported by the formation of stained fat vacuole. The main function of the adipose mesenchymal cell is to produce fat, therefore, it can be a clear evidence to claim that the resulted stem cells are adipose stem cells.

Figure 8 represents the $\mathrm{X}$-ray images of the rabbits' radius after surgery. The dansiometric results of the newly formed bone in week 12 after surgery were reported as $1.45 \pm 0.32$, 1.6 \pm 0.22 , and $0.3 \pm 0.1$ for A (i.e. LDH-HA/Gel scaffolds + ASCs), B (i.e. LDH-HA/GEL scaffolds) and $C$ (i.e. $C T R L$ ), respectively. The difference between groups $A$ and $B$ and control are significant $(\mathrm{P}<0.05)$.

The newly formed high density tissue (i.e. hard callus) mostly filled the defect site in both scaffold groups at week 12 post-implantation, and its amount within the defect site was superior in group A compared with group B (figure 8$)(P<0.05)$. Therefore, it can be concluded that the scaffold dramatically increases the density of the newly formed bone at the critical defect site. In addition, the presence of ASCs accelerates and improves the osteogenesis. This is because the difference of scaffold containing groups with the control group is larger than the difference between the scaffold with ASCs and without them. 
Moreover, the macroscopic images of defect healing confirmed the differences of each group. As it was expected, group A with scaffolds and ASCs showed the most repair and the control group had the lowest repair.

Figure 8 represents the slides of transverse sections of samples after staining with Hematoxylin and Eosin (H\&E) to study the general architecture of the defect area. The cell nucleus and other negatively charged organelles are stained by the hematoxylin (blue) while the cell cytoplasm and most connective tissue containing collagen are stained by the eosin (red/pink).

According to the structure-property relation, the LDH-HA powder showed a better capacity of ion exchange and also has more voids in the nanostructure based on XRD and SEM results. After the scaffolding process, more $\mathrm{H}$-bonds were reported and afterward more swelling occurred. Then the bioactivity evaluation showed ideal secondary HA growth on the scaffold surface. All of these lead to better cellular response and cell-surface interaction and better specific activity of bone such as the ALP level. The final ring of this chain has been observed in the animal test. As shown in figure 8, the scaffold with ASCs was degraded more than the scaffold without cells during 12 weeks. New bone tissue was grown and replaced the degrading scaffold and firmly integrated with the old bone. The cells were proliferated in the newly formed bone while they contained lacunar spaces and were integrated with the periosteum. Also, there is no significant histological evidence of spontaneous bone repair observed in the control sample (group $\mathrm{C}$ ). The quantitative result obtained from the histomorphometric assessment of the area of bone in-growth into the defect indicated that the percentage of bone in-growth for scaffold+ASC $(97.19 \pm 0.17 \%)$ was significantly more prominent than alternate groups $(P<0.05)$. The details of histomorphometric analysis were represented in table 3 . As it is shown in figure 8 , the more mature osteoblasts can be seen in this scaffold compared to the control sample. Also, the more bone mesenchymal cells in the scaffold sample indicate the healing capacity of this scaffold without ASCs. Confirming the previous results, this scaffold could motivate the osteoblast migration and the enormous presence of osteocytes in group B can be observed after 12 weeks. Moreover, the traces of fibrosis tissue after scaffold degradation can be a result of immune system reaction, but after 12 weeks these traces of fibrosis tissue verify the start of bone growth and integrity of the healing process without inflammatory response, confirming the overall health of animals[54].

Obviously, the presence of the scaffold (group B) significantly enhanced the level of bone ingrowth in the critical defect with a full reconstruction of the lost bone that proves the osteoconductivity of the LDH-HA/GEL scaffolds. Also, as mentioned above, the differentiation of ASCs to osteoblast in group B indicates the osteoinductivity of this scaffold.

According to table 3 , the scaffolds of groups A and B showed more than $90 \%$ in vivo degradation after 12 weeks, that were replaced largely by the newly formed bone. Therefore, the degradation of scaffolds occurred in an appropriate rate compared with the new bone formation, therefore the mechanical support will remain until completing the 
healing process that makes this construct suitable for load bearing defects. Eventually, the in vivo results further confirmed the expectations in previous parts. Combining the in vivo outcomes with in vitro results, illustrates the role of $\mathrm{HA}$ in bone healing enhancement in vivo because of its advancing ASC proliferation and osteogenic differentiation ability. Furthermore, the LDH phase has the ability of Ca exchange in the physical environment, which would have a micro scale environment where calcium and phosphate ions can be supersaturated, leading to promotion of bone mineralization. In addition, it can be stated that both enzymatic and hydrolytic degradation mechanisms occurred in vivo environment. In bone histology, osteoid is the organic non-mineralized part of the bone matrix that forms before the bone tissue maturation. Osteoblasts start the procedure of new bone formation by synthesis of osteoid through the expression of specific proteins. While the osteoid gets to be mineralized, it and its surrounding cells have developed into new bone tissue [55]. There is no significant difference between the amounts of the trapped osteoid in the pores of degrading scaffold of the groups $A$ and $B(P>0.05)$. While, in the control sample the amount of osteoid cannot be tracked due to the lack of any supportive matrix to trap or deposit this basic material. Thus, in this case the role of the scaffold composition can be highlighted more than the presence of ASCs. On the other hand, it can be stated here that the degradation process of scaffolds is a result of both hydrolytic and enzymatic mechanisms. Hence, we can conclude that the presence of ASCs in the LDH-HA/GEL scaffold with the novel combination that profits from each component including the LDH ion exchange capacity, the optimized GEL swelling as a bed and the biological patterning role of HA, lead to great in vitro results and the appropriate host response and positively influence the regeneration of the critical defect and segmental fractions. According to the abovementioned data, it seems that using the LDH-HA/GEL scaffold is a promising approach to treat non-union fractures via tissue engineering and cell therapy techniques through mimicking the natural bone structure and properties.

\section{Conclusion}

In the present study, first we synthesized LDH and LDH-HA composite using the wet chemical co-precipitation method, in defined stoichiometric ratios. Then LDH/GEL and LDHHA/GEL composite scaffolds were successfully fabricated by solvent casting the combined lamination method. The results showed the LDH-HA/GEL scaffold with the porosity of $92.11 \pm 0.15 \%$ and Young's modulus of $12.5 \mathrm{GPa}$ and interconnected pores, demonstrating an appropriate bioactivity and degradation behavior. According to the in vitro results, including cell viability, ALP activity and ARS, the cellular response illustrated the biocompatibility of this scaffold. On the other hand, the in vivo results confirmed all of the previous results: both implanted groups (acellular and cell seeded groups) did not show serious inflammatory response and provided new bone formation. The scaffold+cell group allowed more efficient osteogenesis at the defect site, and more precisely, we can say the ASCs dramatically enhance the new bone formation. It should be noted that the in vivo results of the acellular scaffolds investigate the appropriate physiochemical nature of this scaffold for BTE applications. Therefore, the LDH-HA/GEL scaffold has the potential to be a good candidate 
for orthopedic or regenerative surgery. As a result, it seems that this scaffold satisfies the essential necessities of BTE and has the potential to be used in orthopedic and reconstructive surgery.

\section{Acknowledgement}

This work was financially supported by the Iran National Science Foundation (INSF) grant number 88002447 . The authors wish to appreciate Mr. Morteza Mehrjoo (National Cell Bank of Iran) for his help in implementation of the in vitro tests, Dr Farzane Khani (Department of Surgery and Radiology, Faculty of Veterinary Medicine, University of Tehran) for her invaluable contribution in animal tests and Ms. Afsane Vosough (the supervisor of Clinical Research Lab, Endocrinology and Metabolism Clinical Sciences Institute) for her help in obtaining precise results.

\section{Reference}

[1] Woodruff MA, Lange C, Reichert J, Berner A, Chen F, Fratzl P, et al. Bone tissue engineering: from bench to bedside. Materials Today 2012;15:430-5.

[2] Fratzl P, Gupta H, Paschalis E, Roschger P. Structure and mechanical quality of the collagenmineral nano-composite in bone. Journal of materials chemistry 2004;14:2115-23.

[3] Bhatnagar RS, Li S. Biomimetic scaffolds for tissue engineering. Engineering in Medicine and Biology Society, 2004 IEMBS'04 26th Annual International Conference of the IEEE: IEEE; 2004. p. 5021-3.

[4] Ramalingam M, Wang X, Chen G, Ma P, Cui F-Z. Biomimetics: Advancing Nanobiomaterials and Tissue Engineering: John Wiley \& Sons; 2013.

[5] Henkel J, Woodruff MA, Epari DR, Steck R, Glatt V, Dickinson IC, et al. Bone Regeneration Based on Tissue Engineering Conceptions-A 21st Century Perspective. Bone Research 2013;1:216.

[6] Rezwan K, Chen Q, Blaker J, Boccaccini AR. Biodegradable and bioactive porous polymer/inorganic composite scaffolds for bone tissue engineering. Biomaterials 2006;27:3413-31.

[7] Hedberg EL, Shih CK, Lemoine JJ, Timmer MD, Liebschner MA, Jansen JA, et al. In vitro degradation of porous poly (propylene fumarate)/poly (DL-lactic-co-glycolic acid) composite scaffolds. Biomaterials 2005;26:3215-25.

[8] Schieker M, Seitz H, Drosse I, Seitz S, Mutschler W. Biomaterials as scaffold for bone tissue engineering. European journal of trauma 2006;32:114-24.

[9] Salgado AJ, Coutinho OP, Reis RL. Bone tissue engineering: state of the art and future trends. Macromolecular bioscience 2004;4:743-65.

[10] El-Ghannam A. Bone reconstruction: from bioceramics to tissue engineering. Expert review of medical devices 2005;2:87-101.

[11] Hench LL. The story of Bioglass ${ }^{\circledR}$. Journal of Materials Science: Materials in Medicine 2006;17:967-78.

[12] LeGeros RZ. Properties of osteoconductive biomaterials: calcium phosphates. Clinical orthopaedics and related research 2002;395:81-98.

[13] Gómez-Guillén M, Giménez B, López-Caballero Ma, Montero M. Functional and bioactive properties of collagen and gelatin from alternative sources: A review. Food Hydrocolloids 2011;25:1813-27. 
[14] Gashti MP, Bourquin M, Stir M, Hulliger J. Glutamic acid inducing kidney stone biomimicry by a brushite/gelatin composite. Journal of Materials Chemistry B 2013;1:1501-8.

[15] Gashti MP, Burgener M, Stir M, Hulliger J. Barium hydrogen phosphate/gelatin composites versus gelatin-free barium hydrogen phosphate: Synthesis and characterization of properties. Journal of colloid and interface science 2014;431:149-56.

[16] Gashti MP, Stir M, Hulliger J. Growth of strontium hydrogen phosphate/gelatin composites: a biomimetic approach. New Journal of Chemistry 2016;40:5495-500.

[17] Azami M, Tavakol S, Samadikuchaksaraei A, Hashjin MS, Baheiraei N, Kamali M, et al. A porous hydroxyapatite/gelatin nanocomposite scaffold for bone tissue repair: in vitro and in vivo evaluation. Journal of Biomaterials Science, Polymer Edition 2012;23:2353-68.

[18] Duan X, Evans DG. Layered Double Hydroxides (Structure and Bonding): Springer Science \& Business Media; 2006.

[19] Fayyazbakhsh F, Solati-Hashjin M, Shokrgozar M, Bonakdar S, Ganji Y, Mirjordavi N, et al. Biological Evaluation of a Novel Tissue Engineering Scaffold of Layered Double Hydroxides (LDHs). Key Engineering Materials: Trans Tech Publ; 2012. p. 902-8.

[20] Shafiei SS, Solati-Hashjin M, Rahim-Zadeh H, Samadikuchaksaraei A. Synthesis and characterisation of nanocrystalline Ca-Al layered double hydroxide $\{[\mathrm{Ca} 2 \mathrm{Al}(\mathrm{OH}) 6] \mathrm{NO}$. $\mathrm{nH} 2 \mathrm{O}\}$ : in vitro study. Advances in Applied Ceramics 2013;112:59-65.

[21] Rabiee S, Moztarzadeh F, Solati-Hashjin M. Synthesis and characterization of hydroxyapatite cement. Journal of Molecular Structure 2010;969:172-5.

[22] Kim HW, Knowles JC, Kim HE. Hydroxyapatite and gelatin composite foams processed via novel freeze-drying and crosslinking for use as temporary hard tissue scaffolds. Journal of Biomedical Materials Research Part A 2005;72:136-45.

[23] Kokubo T, Takadama H. How useful is SBF in predicting in vivo bone bioactivity? Biomaterials 2006;27:2907-15.

[24] Lucero CM, Vega OA, Osorio MM, Tapia JC, Antonelli M, Stein GS, et al. The cancer-related transcription factor Runx2 modulates cell proliferation in human osteosarcoma cell lines. Journal of cellular physiology 2013;228:714-23.

[25] Lee RH, Kim B, Choi I, Kim H, Choi H, Suh K, et al. Characterization and expression analysis of mesenchymal stem cells from human bone marrow and adipose tissue. Cellular Physiology and Biochemistry 2004;14:311-24.

[26] Murugan R, Ramakrishna S. Crystallographic study of hydroxyapatite bioceramics derived from various sources. Crystal growth \& design 2005;5:111-2.

[27] Miyata S. The syntheses of hydrotalcite-like compounds and their structures and physicochemical properties I: The systems Mg2+-Al3+-NO3-, Mg2+-Al3+-Cl-, Mg2+-Al3+-ClO4-, Ni2+-Al3+-Cland Zn2+-Al3+-Cl. Clays and Clay Minerals 1975;23:369-75.

[28] Ślósarczyk A, Paszkiewicz Z, Paluszkiewicz C. FTIR and XRD evaluation of carbonated hydroxyapatite powders synthesized by wet methods. Journal of Molecular Structure 2005;744:65761.

[29] Hattori T, Lwadate Y. Hydrothermal preparation of calcium hydroxyapatite powders. Journal of the American Ceramic Society 1990;73:1803-5.

[30] Koutsopoulos S. Synthesis and characterization of hydroxyapatite crystals: a review study on the analytical methods. Journal of biomedical materials research 2002;62:600-12.

[31] Khan Al, O'Hare D. Intercalation chemistry of layered double hydroxides: recent developments and applications. Journal of Materials Chemistry 2002;12:3191-8. 
[32] Berzina-Cimdina L, Borodajenko N. Research of calcium phosphates using Fourier transform infrared spectroscopy: INTECH Open Access Publisher; 2012.

[33] Tao Q, Reddy BJ, He H, Frost RL, Yuan P, Zhu J. Synthesis and infrared spectroscopic characterization of selected layered double hydroxides containing divalent $\mathrm{Ni}$ and $\mathrm{Co}$. Materials Chemistry and Physics 2008;112:869-75.

[34] Muyonga J, Cole C, Duodu K. Fourier transform infrared (FTIR) spectroscopic study of acid soluble collagen and gelatin from skins and bones of young and adult Nile perch (Lates niloticus). Food Chemistry 2004;86:325-32.

[35] Kazemzadeh Narbat M, Orang F, Solati Hashtjin M, Goudarzi A. Fabrication of porous hydroxyapatite-gelatin composite scaffolds for bone tissue engineering. Iranian Biomedical Journal 2006;10:215-23.

[36] Tanaka T, Kameshima Y, Nishimoto S, Miyake M. Determination of carbonate ion contents in layered double hydroxides by FTIR spectrometry. Analytical Methods 2012;4:3925-7.

[37] Chang MC, Tanaka J. FT-IR study for hydroxyapatite/collagen nanocomposite cross-linked by glutaraldehyde. Biomaterials 2002;23:4811-8.

[38] Socrates G. Infrared and Raman characteristic group frequencies: tables and charts: John Wiley \& Sons; 2004.

[39] Fürst W, Banerjee A. Release of glutaraldehyde from an albumin-glutaraldehyde tissue adhesive causes significant in vitro and in vivo toxicity. The Annals of thoracic surgery 2005;79:1522-8.

[40] Zhu H, Narakathu BB, Fang Z, Aijazi AT, Joyce M, Atashbar M, et al. A gravure printed antenna on shape-stable transparent nanopaper. Nanoscale 2014;6:9110-5.

[41] Tripathi G, Basu B. A porous hydroxyapatite scaffold for bone tissue engineering: Physicomechanical and biological evaluations. Ceramics international 2012;38:341-9.

[42] Alger M. Polymer science dictionary: Springer Science \& Business Media; 1996.

[43] Sena LA, Caraballo MM, Rossi AM, Soares GA. Synthesis and characterization of biocomposites with different hydroxyapatite-collagen ratios. Journal of Materials Science: Materials in Medicine 2009;20:2395-400.

[44] Jain E, Srivastava A, Kumar A. Macroporous interpenetrating cryogel network of poly (acrylonitrile) and gelatin for biomedical applications. Journal of Materials Science: Materials in Medicine 2009;20:173-9.

[45] Li-ping L, Yan-bing X, Zi-wen X, Zhi-biao W. Toxicity of hydroxyapatite nanoparticles on rabbits. Journal of Hygiene Research 2005;4:031.

[46] Wang L, Zhou G, Liu H, Niu X, Han J, Zheng L, et al. Nano-hydroxyapatite particles induce apoptosis on MC3T3-E1 cells and tissue cells in SD rats. Nanoscale 2012;4:2894-9.

[47] Li B, Guo B, Fan H, Zhang X. Preparation of nano-hydroxyapatite particles with different morphology and their response to highly malignant melanoma cells in vitro. Applied Surface Science 2008;255:357-60.

[48] Meyer U, Wiesmann HP. Bone and cartilage engineering: Springer Science \& Business Media; 2006.

[49] Wang H, Bongio M, Farbod K, Nijhuis AW, van den Beucken J, Boerman OC, et al. Development of injectable organic/inorganic colloidal composite gels made of self-assembling gelatin nanospheres and calcium phosphate nanocrystals. Acta biomaterialia 2014;10:508-19.

[50] Dong S-W, Ying D-J, Duan X-J, Xie Z, Yu Z-J, Zhu C-H, et al. Bone regeneration using an acellular extracellular matrix and bone marrow mesenchymal stem cells expressing Cbfa1. Bioscience, biotechnology, and biochemistry 2009;73:2226-33. 
[51] Kim H-W, Kim H-E, Salih V. Stimulation of osteoblast responses to biomimetic nanocomposites of gelatin-hydroxyapatite for tissue engineering scaffolds. Biomaterials 2005;26:5221-30.

[52] Dvorak MM, Riccardi D. Ca 2+ as an extracellular signal in bone. Cell calcium 2004;35:249-55.

[53] Chiu C-K, Ferreira J, Luo T-JM, Geng H, Lin F-C, Ko C-C. Direct scaffolding of biomimetic hydroxyapatite-gelatin nanocomposites using aminosilane cross-linker for bone regeneration. Journal of Materials Science: Materials in Medicine 2012;23:2115-26.

[54] Sharma H, Zerbe N, Lohmann S, Kayser K, Hellwich O, Hufnagl P. A review of graph-based methods for image analysis in digital histopathology. Diagnostic Pathology 2015;1.

[55] Morgan EF, Barnes GL, Einhorn TA, Marcus R, Feldman D, Nelson D, et al. The bone organ system: form and function: Academic Press; 2013.

[56] Cristofolini L, Taddei F, Baleani M, Baruffaldi F, Stea S, Viceconti M. Multiscale investigation of the functional properties of the human femur. Philosophical Transactions of the Royal Society of London A: Mathematical, Physical and Engineering Sciences 2008;366:3319-41.

Figure 1- The general implantation process: a- fat tissue sampling from intrascapular area b- OM image of cultured ASCs (magnification 40x), c- measuring and marking the defect site on radius bone, $d$ - cutting the radius to create the defect by surgical saws, e- with/without ASC scaffolds were soaked in culture media and then swelled, the swollen scaffold vs. dissected bone. The size differences can be seen but the swollen scaffold showed a flexible behavior therefore it was placed in the defect site, $\mathrm{f}$ - the scaffold placed in the defect site

(i.e. implanted), g- muscle sutures after implanting, $h$ - dermal sutures, $\mathrm{i}$ - radiography position and the aluminum graded phantom to evaluate the density of newly formed bone

Figure 2- micrographs of specimens: a- micrographs of specimens, b- LDH-HA powder, c- LDH structure (the interaction of LDH and HA has been discussed later), d- Pure LDH/GEL scaffold, e- LDH-HA/GEL scaffold, fnatural bone microstructure for comparison with synthetic scaffolds (high structural similarity can be seen between bone and LDH-HA/GEL interconnected scaffold) (magnification: 100x), g- G-292s on the surface of Pure LDH-GEL scaffold, h- G-292s on the surface of LDH-HA/GEL scaffold, i- interaction between G-292s and

LDH-HA/GEL scaffold (the cellular activity such as adhesion, proliferation and migration can be seen)

Figure 3- (a) XRD pattern of specimens (b) FTIR pattern of scaffold specimens (c) Mechanical properties (the natural spongy bone data were adopted from Cristofolini et al. [56]

Figure 4- a- Weight loss profile of specimens. these plots include two main parts: water absorbance (swelling) and degradation. Dependence of water absorbance and degradation to time. $b$ - The changes of $\mathrm{pH}$ in scaffolds

Figure 5- The SEM images of scaffolds after immersing in SBF. a, b, c: pure LDH/GEL scaffold, s, f, g: LDH$\mathrm{HA} / \mathrm{GEL}$ scaffold with different magnification. a and e: the nucleation of secondary HA through the pores after day $3, \mathrm{~b}$ and f: the growth of secondary HA crystals and scaffold degradation after day $14, \mathrm{c}$, g: needle-like morphology of secondary HA on the surface of scaffolds after day 21 
Figure 6- a- The result of MTT assay for powders' extraction and G-292 cells, the scaffolds showed better result than powders and control sample $(P<0.05)$ after 14 days the amounts of viability for LDH-HA scaffolds and control sample didn't show a meaningful difference $(P>0.05)$ but the $L D H$ HA scaffold is better than powders and pure LDH scaffold $(\mathrm{P}<0.05), *, * *, * * *$ : $(\mathrm{P}<0.05) \mathrm{b}$ - The ALP activity of powder and scaffolds. The LDHHA/GEL scaffold showed better ALP activity *,**,*** $(\mathrm{P}<0.05)$

Figure 7- OM images of scaffolds after Alizarin red staining: a, c, d: LDH/GEL, LDH-HA/GEL, CTRL samples after G-292 seeding, e, f, g: LDH/GEL, LDH-HA/GEL, CTRL samples after rabbit ASC seeding. These images show that the scaffolds can clearly promote calcium mineralization by osteoblast cells or ASCs i: formation of fat vacuole around differentiated stem cells proves that the extracted stem cells' identity is the adipose stem cell.

(magnification for a to $\mathrm{h}$ images: $100 \mathrm{x}$ and for $\mathrm{i}$ image: $400 \mathrm{x}$ ).

Figure 8- First column: The X-rays images of the rabbit radius defects treated with A: scaffold+ASC, B: scaffolds and C: no treatment (control) after 12 weeks post-surgery. The group A showed the highest density of newly formed bone. The lower images indicate the macroscopic new bone formation. The greatest healing (observable to the naked eye) belongs to group A. second column: The integrity of new bone formed with surrounding tissue occurred with a better quality in this sample. Third column: OM images of H\&E staining of transverse bone defect sections at 12 weeks post-surgery in all groups. Both implanted groups ( $A$ and $B$ ) did not provoke any significant inflammatory response or foreign body reaction. Both $A$ and $B$ groups allowed bone in-growth, while the ASC containing group A showed quicker and more viable osteogenesis at the defect site than alternate groups. Abbreviations: scaffold residues (S), new bone (NB), and fibrous tissue (FT). Signs: white arrow: bone mesenchymal cells, black arrow: mature differentiated osteoblasts, yellow arrow: soft callus, green arrow: trace of fibrosis tissue transforming to bone tissue Scale bars: $50 \mu \mathrm{m}$ 
Table 1- The lattice parameters and LDH/HA ratio for powder samples. As it is shown, the lattice parameter for the HA phase remained constant and for the LDH phase, they were changed and RIR confirmed the synthesis of powders (the prediction of ratio), as it confirmed by EDS results.

\begin{tabular}{|c|c|c|}
\hline Specimen & LDH & LDH-HA \\
\hline RIR (LDH to HA) & - & $76 \%$ \\
\hline Lattice parameter a $(\AA)$ for LDH phase & 3.021095 & 3.053048 \\
\hline Lattice parameter c $(\AA \AA)$ for LDH phase & 16.45157 & 15.66154 \\
\hline Lattice parameter a $(\AA)$ for HA phase & - & 9.521861 \\
\hline Lattice parameter c $(\AA)$ for HA phase & - & 6.981945 \\
\hline $\mathrm{Ca} / \mathrm{P}$ & - & 7.65 \\
\hline $\mathrm{Ca} / \mathrm{Al}$ & 2.02 & 2.54 \\
\hline
\end{tabular}

Table 2- Physiomechanical properties of scaffolds VS. natural spongy/cortical bone $[72,76,77]$

\begin{tabular}{|c|c|c|c|c|c|c|c|}
\hline Scaffold & $\begin{array}{c}\rho \\
\left(\mathrm{g} / \mathrm{cm}^{3}\right)\end{array}$ & $\begin{array}{c}\mathrm{E} \\
(\mathrm{GPa})\end{array}$ & $\begin{array}{c}\text { Stiffness } \\
(\mathrm{N} / \mathrm{mm})\end{array}$ & $\begin{array}{c}\text { 6yield } \\
(\mathrm{kN})\end{array}$ & $\begin{array}{c}\text { Porosity } \\
(\%)\end{array}$ & $\begin{array}{c}\text { Pore size } \\
(\mu \mathrm{m})\end{array}$ & $\begin{array}{c}\text { Wall } \\
\text { thickness } \\
(\mu \mathrm{m})\end{array}$ \\
\hline $\mathrm{LDH} / \mathrm{GEL}$ & $0.284 \pm 0.019$ & $19.8 \pm 0.41$ & 228 & $2.3 \pm 0.5$ & $90.0 \pm 0.12$ & $200-600$ & $20-40$ \\
\hline LDH-HA/GEL & $0.279 \pm 0.031$ & $12.5 \pm 0.35$ & 186 & $1.9 \pm 0.5$ & $92.11 \pm 0.15$ & $100-300$ & $20-40$ \\
\hline $\begin{array}{c}\text { Natural } \\
\text { spongy bone }\end{array}$ & $0.14-1.2$ & $0.02-0.5$ & & $4-12$ & $30-90$ & $100-900$ & $1-50$ \\
\hline $\begin{array}{c}\text { Natural } \\
\text { cortical bone }\end{array}$ & $1.8-2$ & $3-30$ & & $130-180$ & $5-30$ & $0.1-1$ & $<100 \mathrm{~nm}$ \\
\hline
\end{tabular}

Table 3- Histomorphometric quantitative results in week 12

\begin{tabular}{|c|c|c|c|c|c|c|c|}
\hline \multicolumn{1}{|c|}{ Sample } & Densitometry & $\begin{array}{c}\text { Cortical } \\
\text { bone }\end{array}$ & $\begin{array}{c}\text { Trabecular } \\
\text { bone }\end{array}$ & $\begin{array}{c}\text { Connective } \\
\text { tissue }\end{array}$ & $\begin{array}{c}\text { Scaffold } \\
\text { residues }\end{array}$ & Osteoid & Cavity \\
\hline $\begin{array}{c}\text { LDH-HA/GEL } \\
+ \text { ASC }\end{array}$ & $1.6 \pm 0.22$ & $69.9 \pm 3.8$ & $10.6 \pm 5.6$ & $2.8 \pm 0.7$ & $6.8 \pm 1.6$ & $2.8 \pm 0.8$ & $7.2 \pm 1.6$ \\
\hline LDH-HA/GEL & $1.45 \pm 0.32$ & $61.2 \pm 5.5$ & $7.1 \pm 2.7$ & $2.8 \pm 0.8$ & $8.6 \pm 3.7$ & $2.5 \pm 1.07$ & $17.8 \pm 3.4$ \\
\hline CTRL & $0.2 \pm 0.1$ & $1.08 \pm 0.1$ & $0.4 \pm 0.1$ & $42.1 \pm 7.7$ & - & - & $56.3 \pm 7.8$ \\
\hline
\end{tabular}



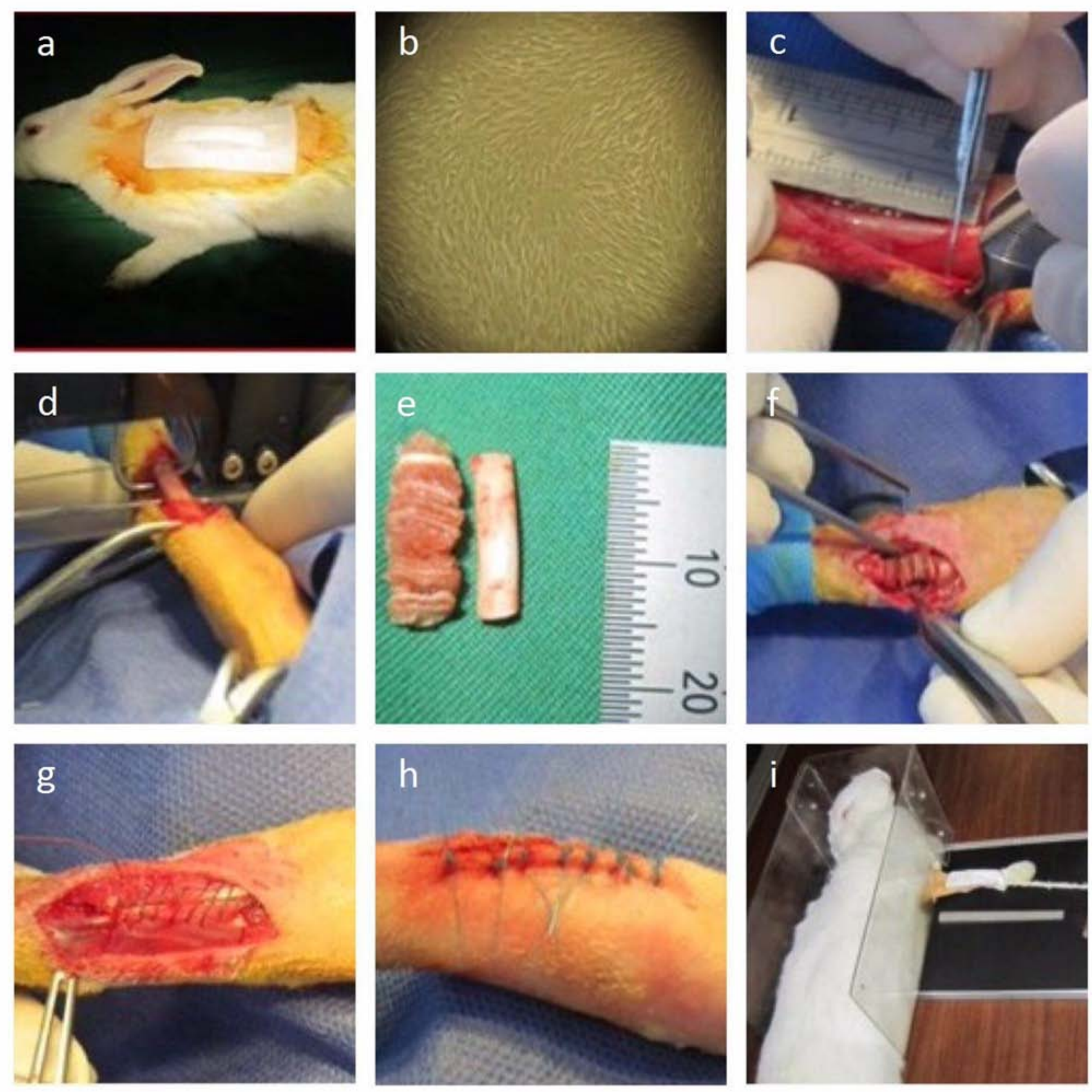

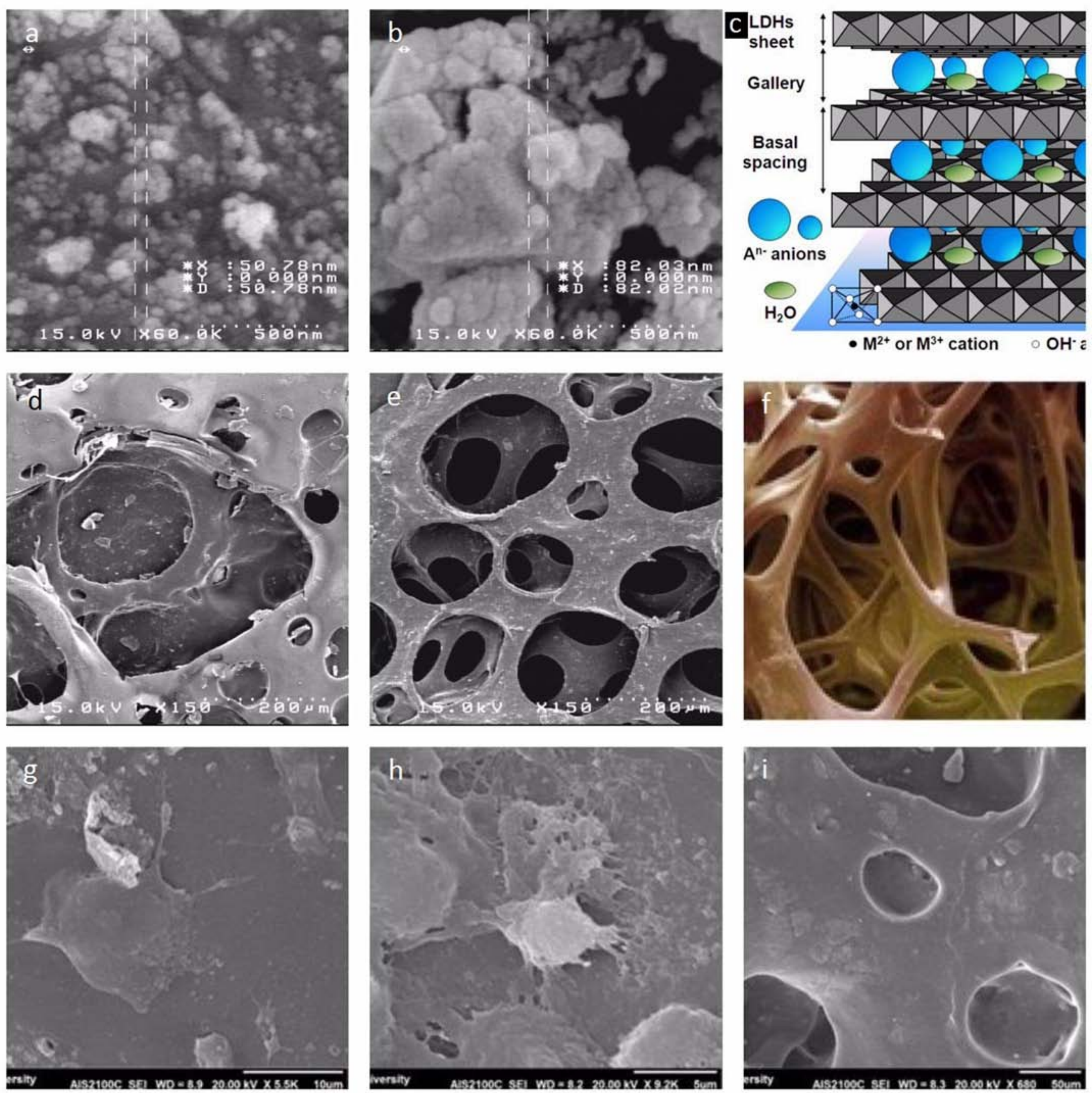
(a)

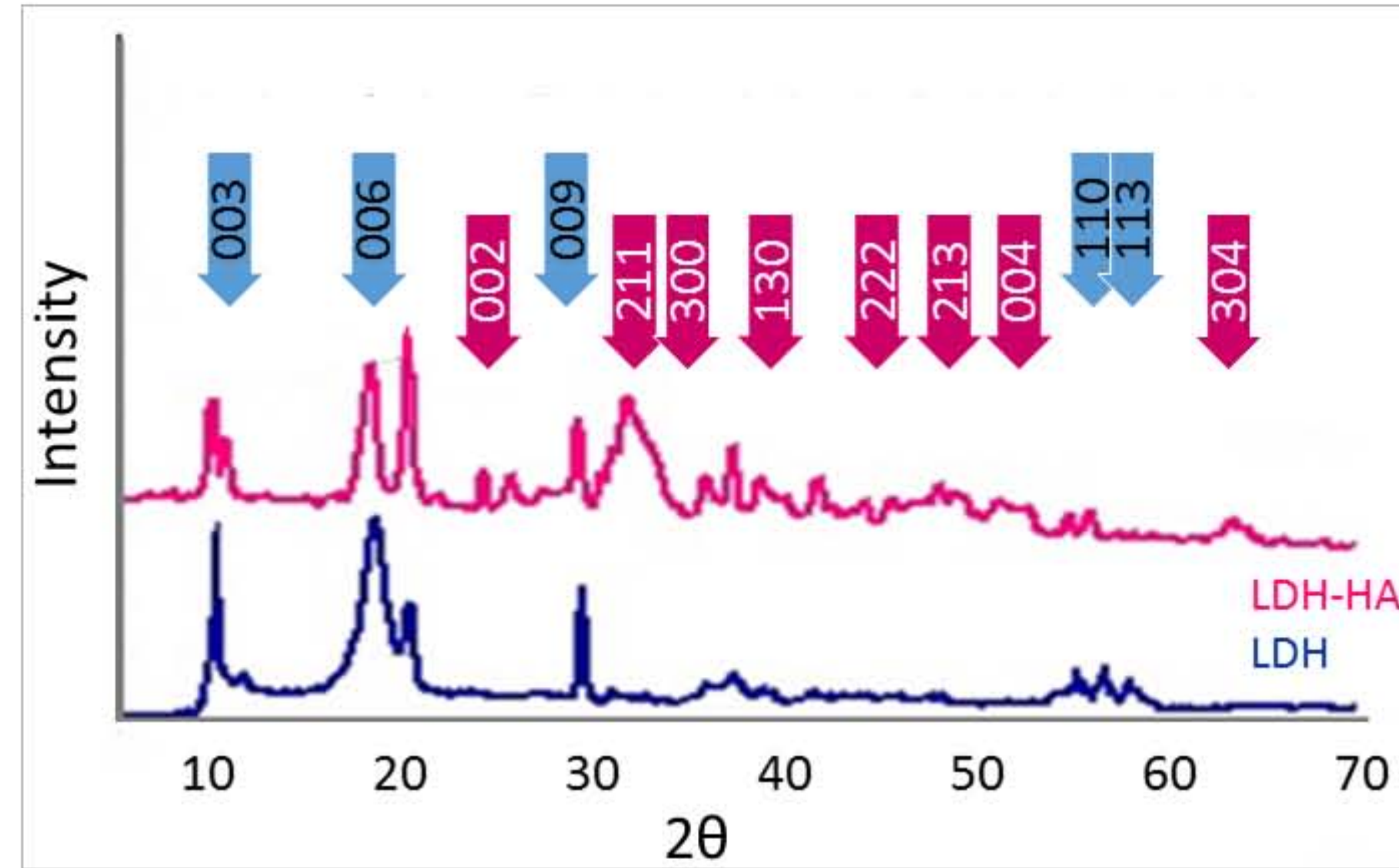

LDH/GEL scaffold LDH-HA/GEL scaffold

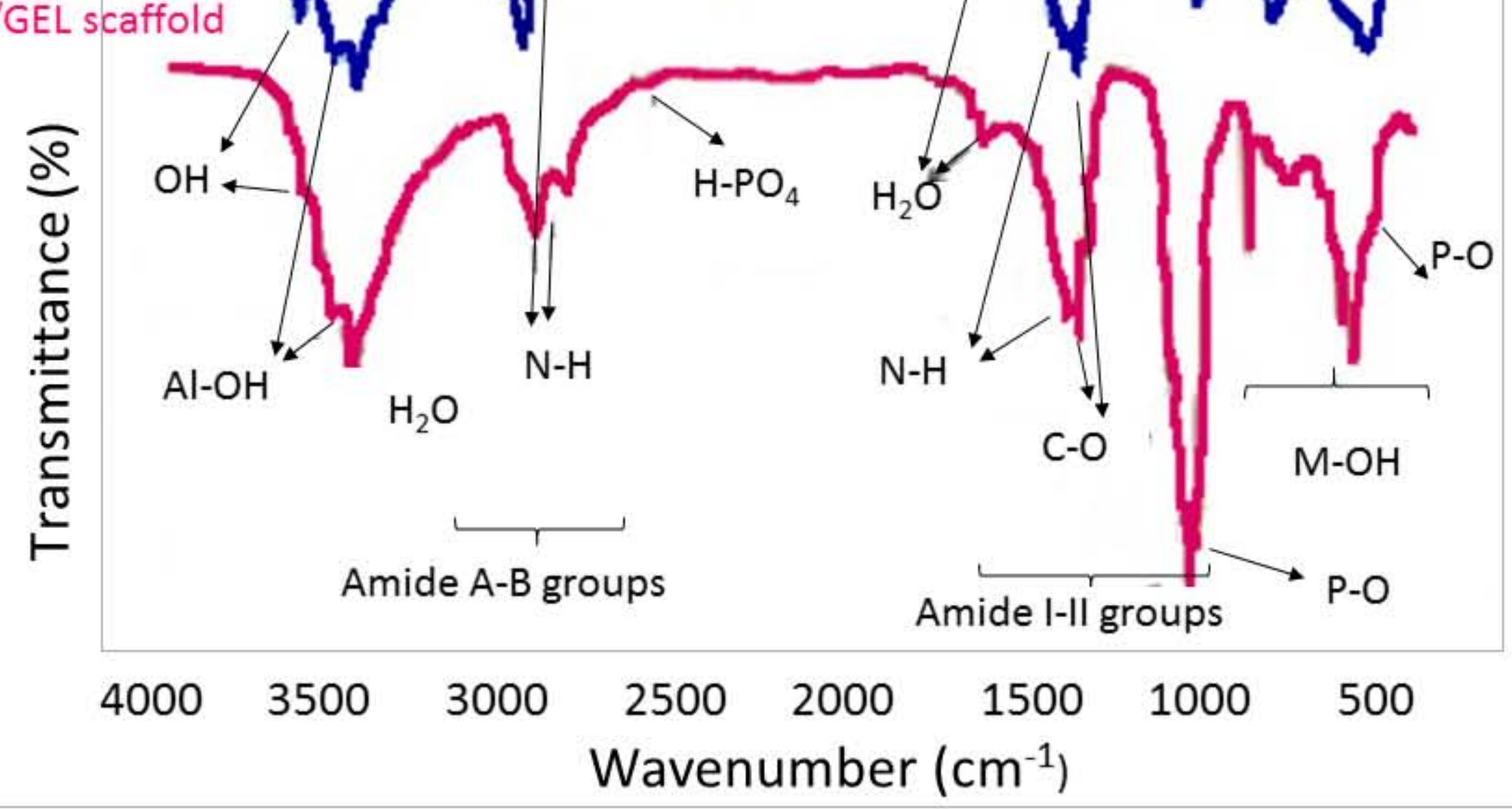

(c)

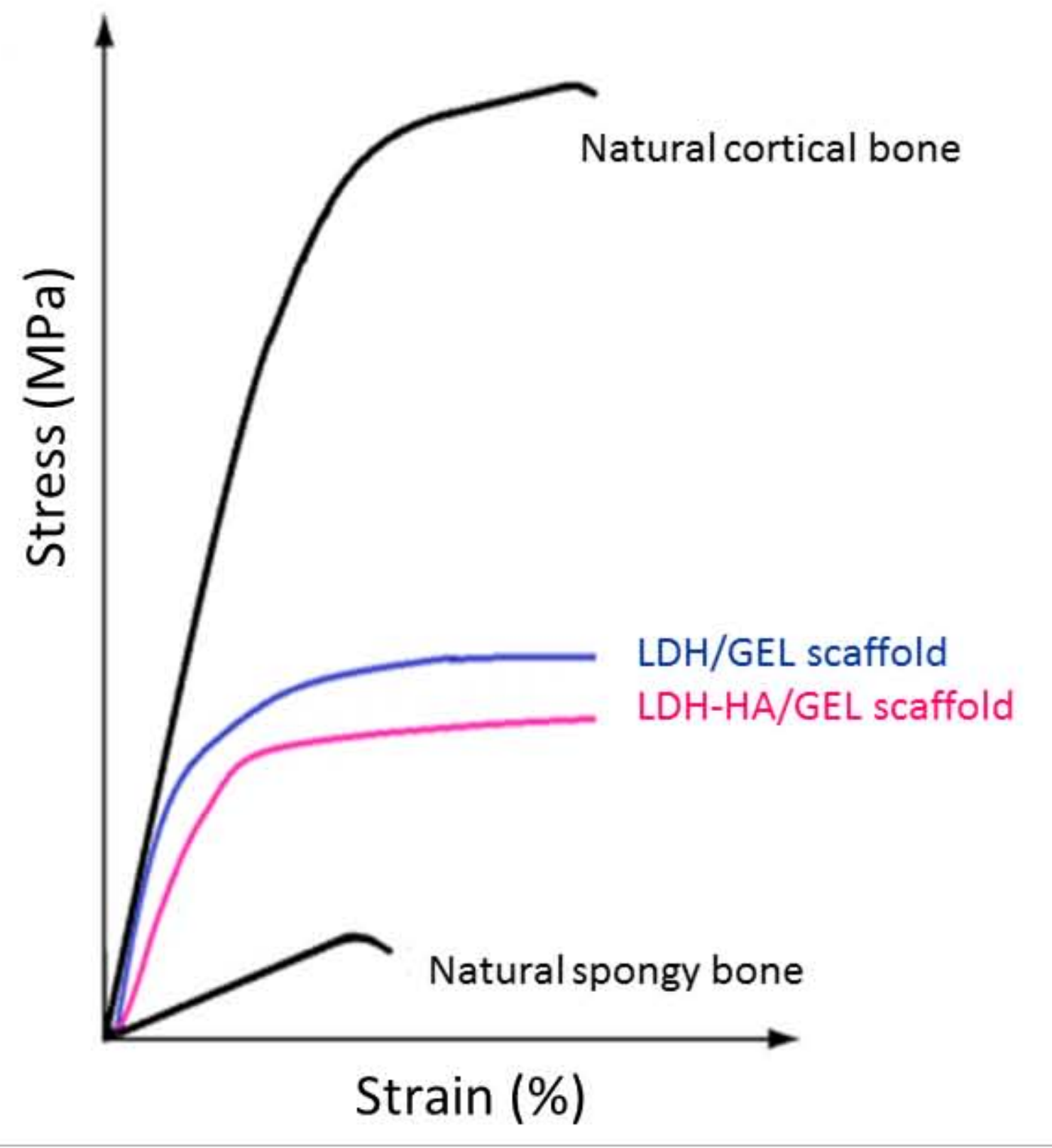



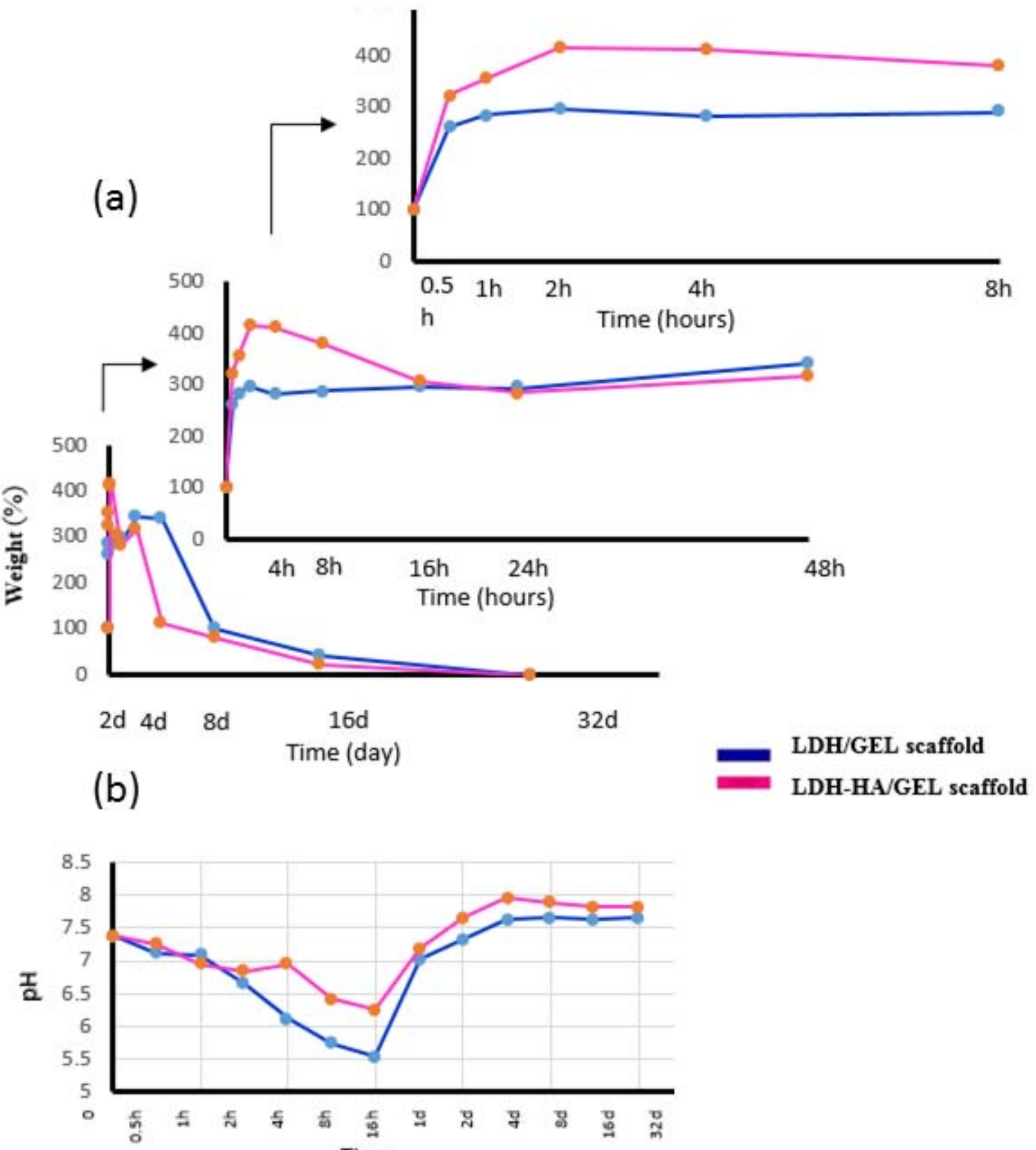

Time 


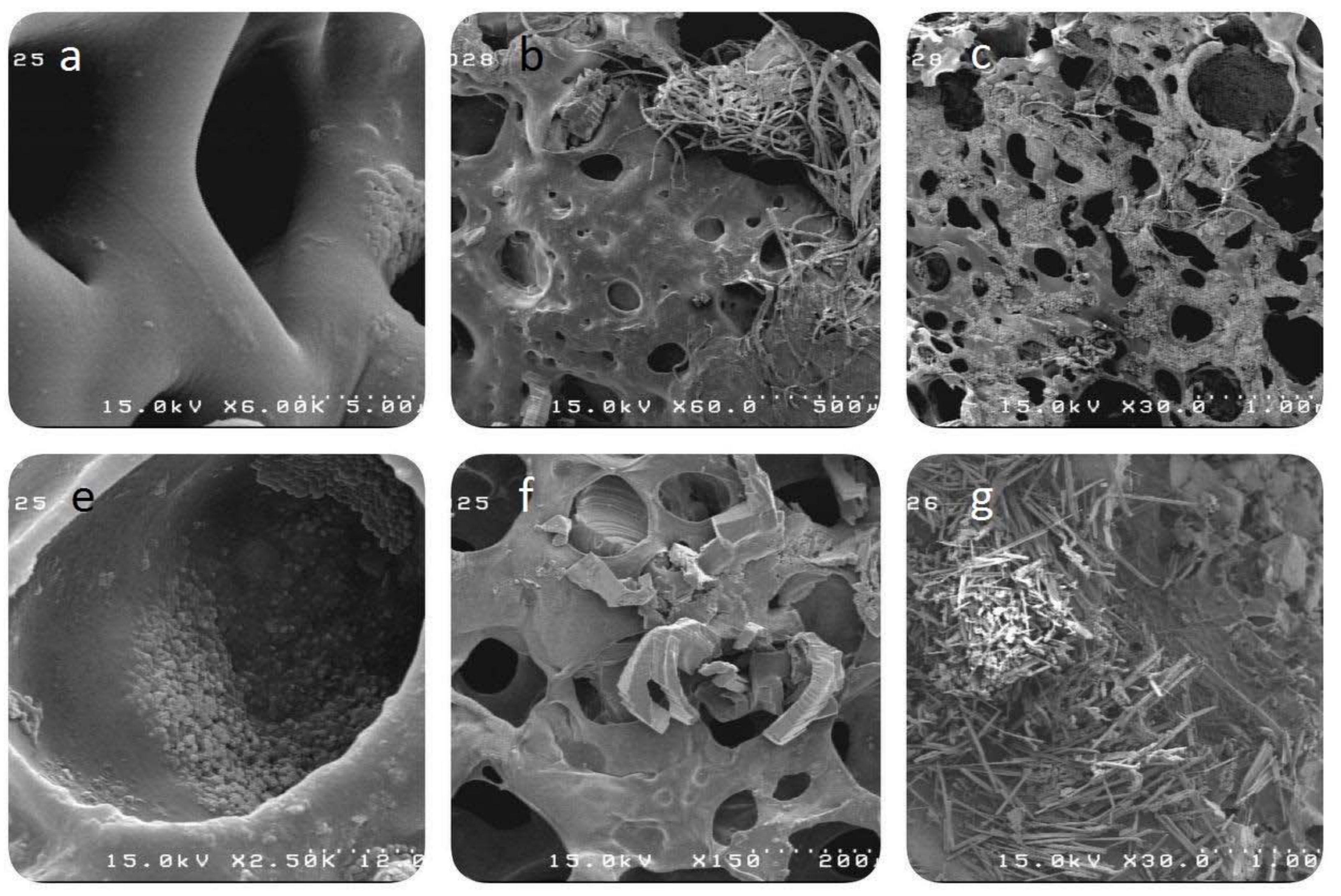


(a)

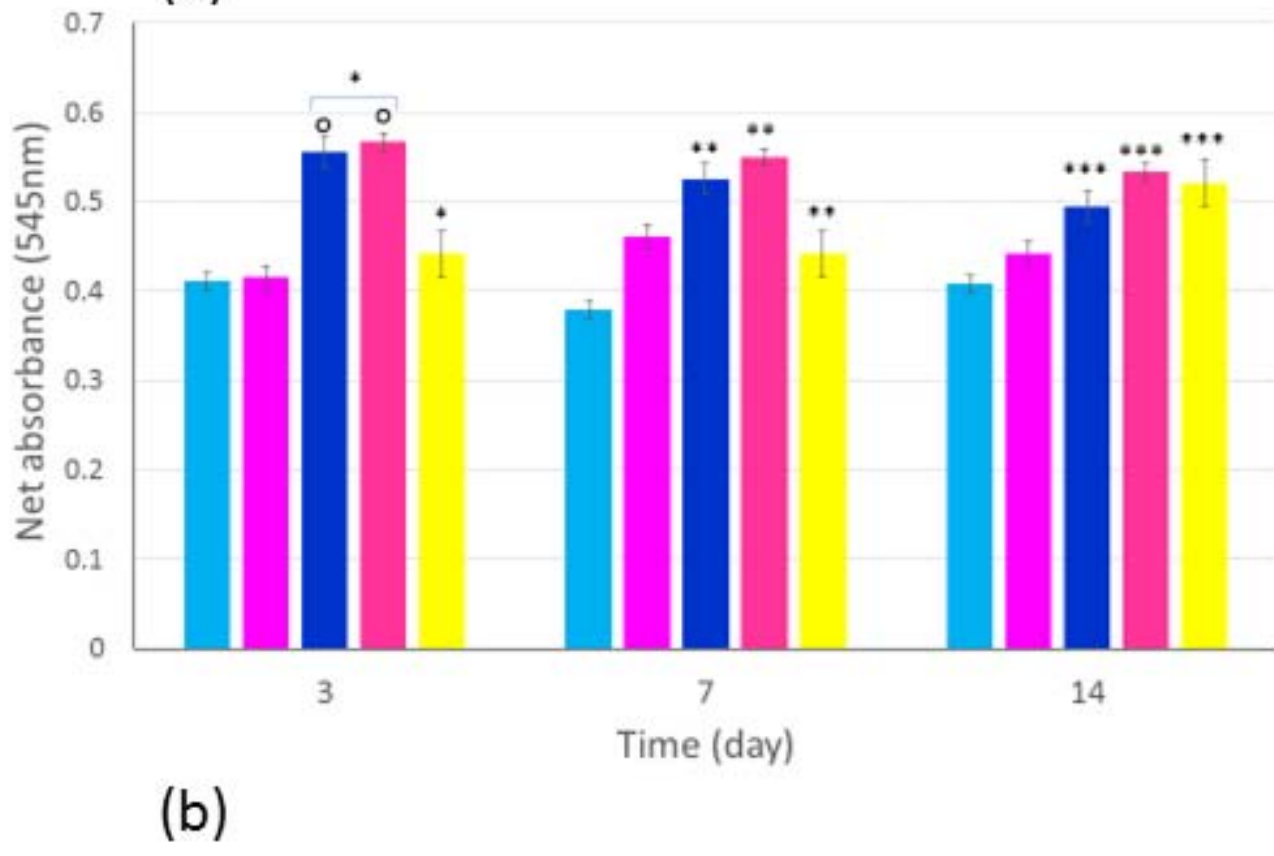

a LDH powder

- LDH-HA powder

- LDH/GEL scaffold

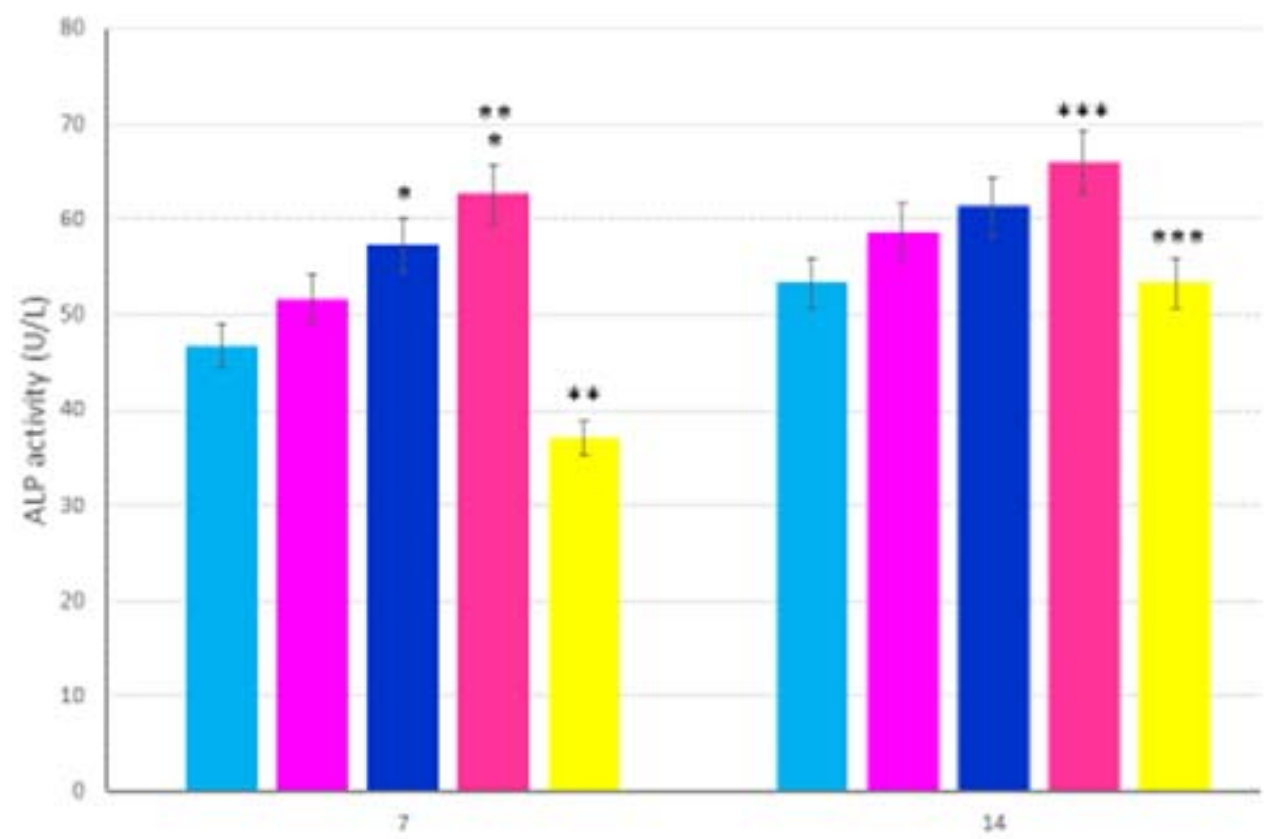

a LDH-HA/GEL scaffold

CTRL (Culture media) 

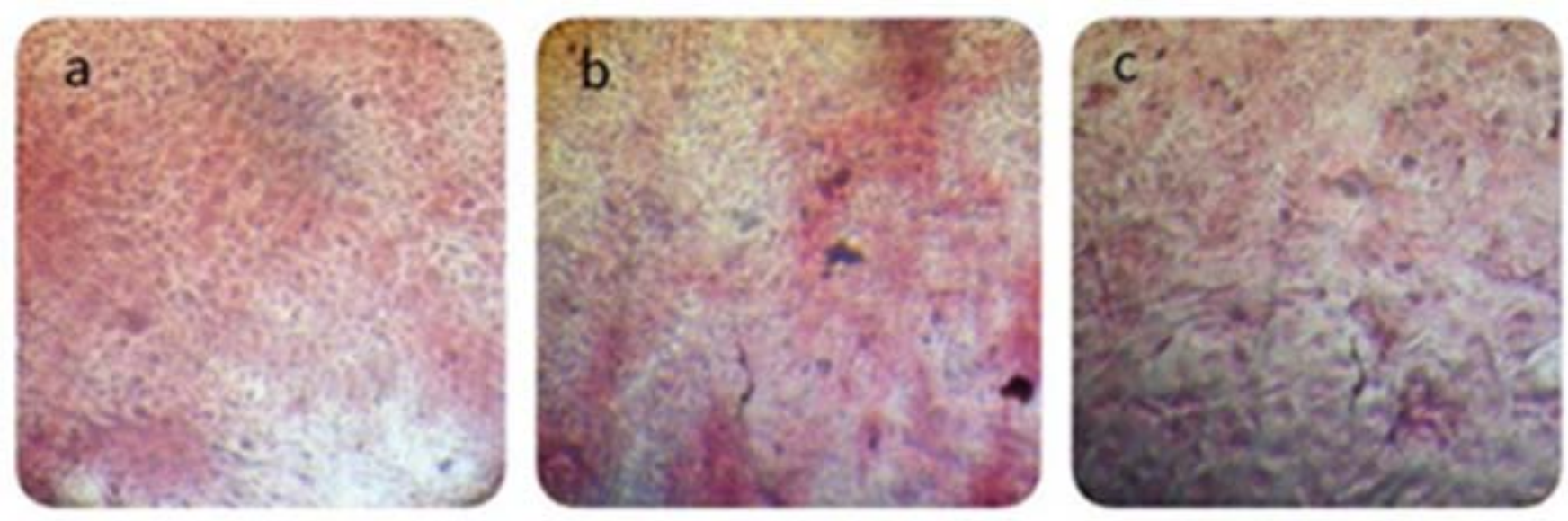

\section{e}
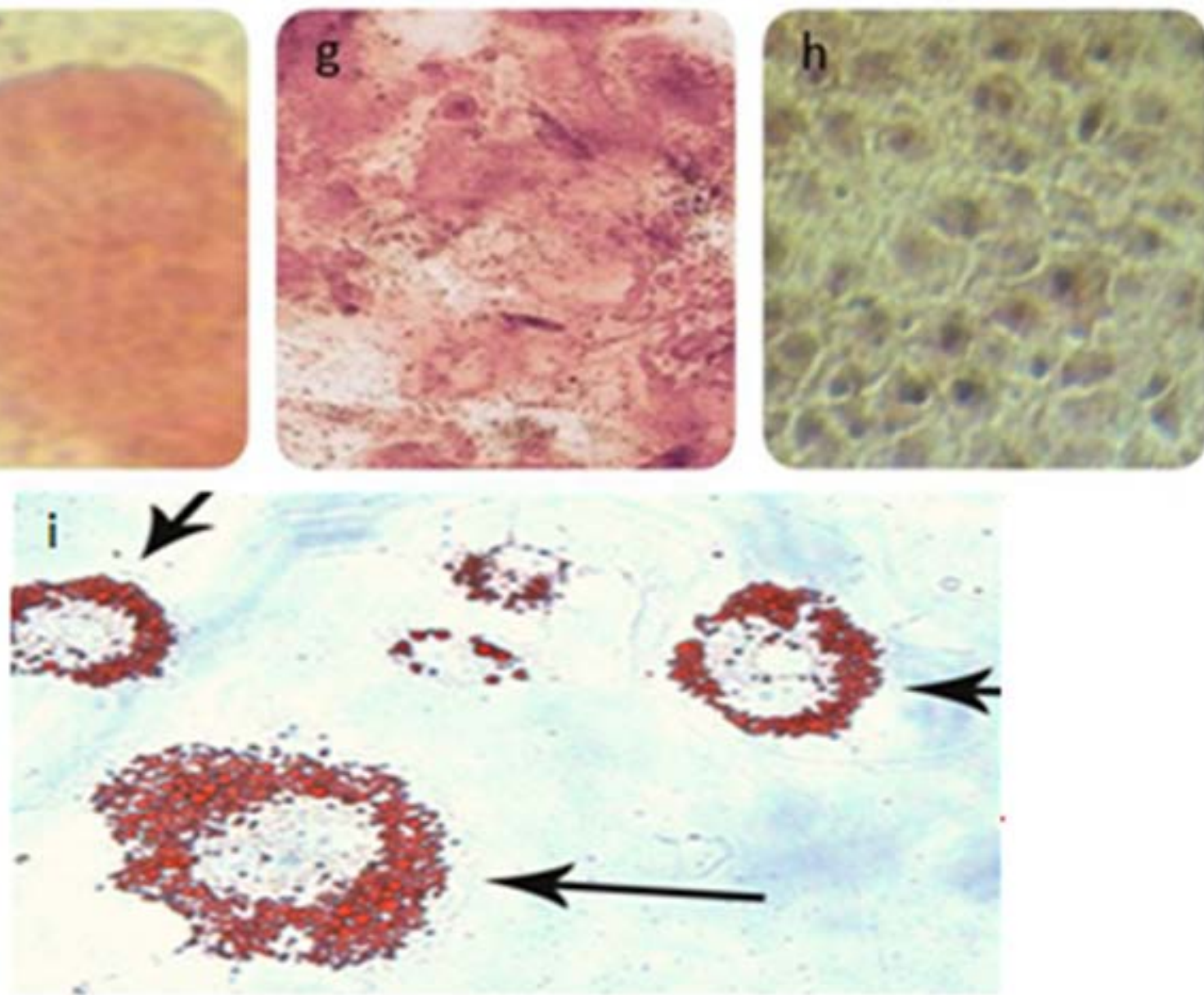

. 

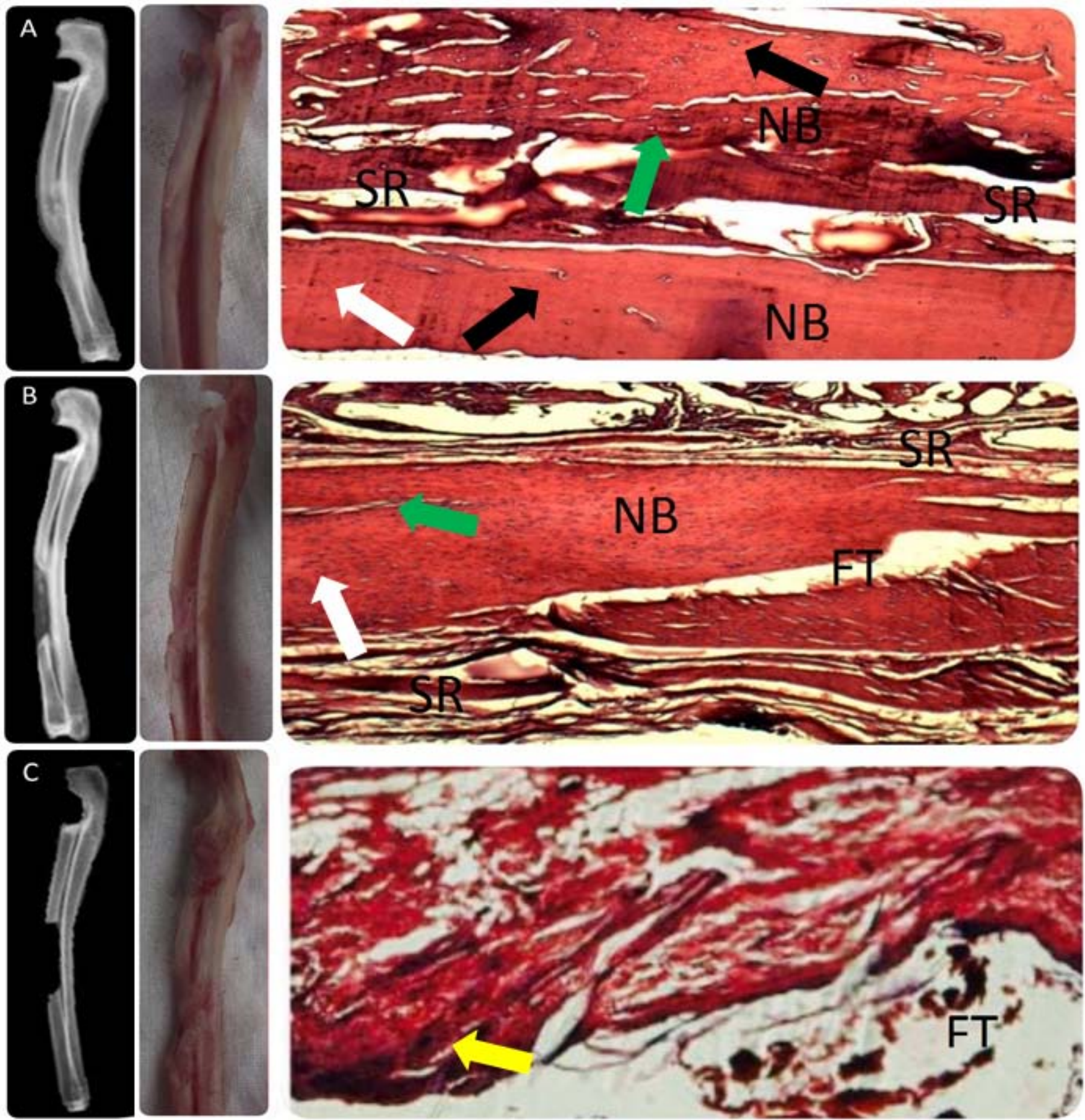
C C AT

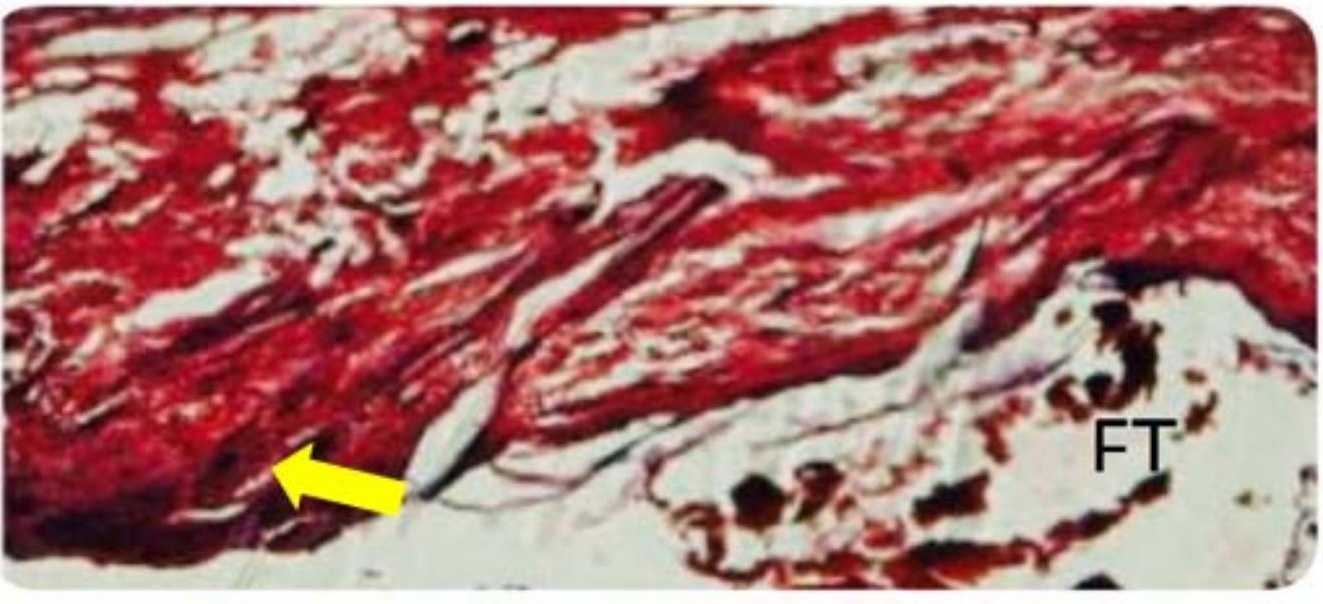


Mesenchymal

Adipose Stem Cells

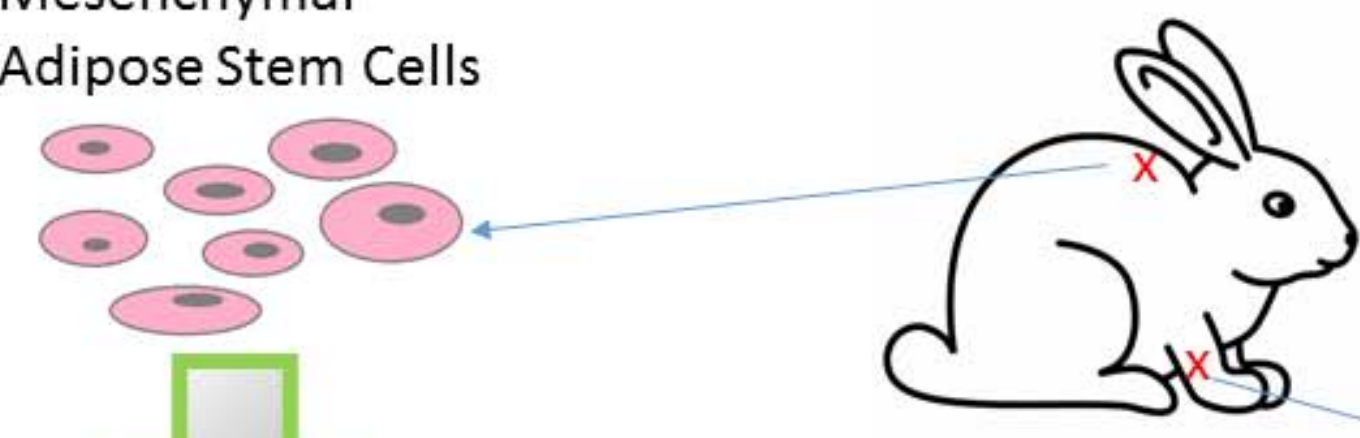

Porous Layered Scaffold

In vivo Implantation

Gelatin Chains

Layered Double

Hydroxides Sheets

Hydroxyapatite

Appropriate Diffusion,

Degradation rate and mechanical properties lead to accelerate the regeneration process

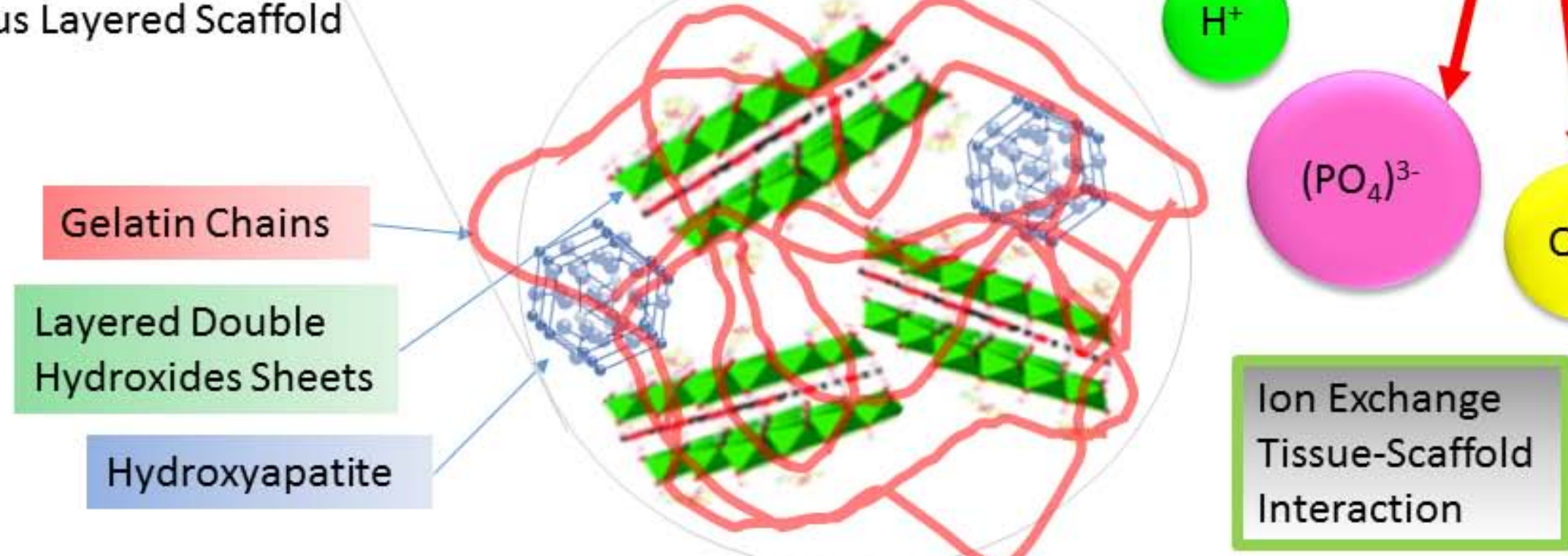

Bioactive Surface

Secondary HA formation 